\title{
Reactivity of allenylphosphonates and allenylphosphine oxides toward 9-chloroacridines and acridone- A facile route to new $N$-substituted acridones
}

\author{
A LEELA SIVA KUMARI, VENU SRINIVAS and K C KUMARA SWAMY* \\ School of Chemistry, University of Hyderabad, Hyderabad 500 046, India \\ e-mail: kckssc@uohyd.ac.in; kckssc@yahoo.com
}

MS received 4 June 2013; revised 16 August 2013; accepted 21 August 2013

\begin{abstract}
Base-mediated addition of acridones to allenylphosphonates/allenylphosphine oxides $\left(\mathrm{OCH}_{2} \mathrm{CMe}_{2} \mathrm{CH}_{2} \mathrm{O}\right) \mathrm{P}(\mathrm{O}) \mathrm{CH}=\mathrm{C}=\mathrm{CR}^{1} \mathrm{R}^{2}\left\{\mathrm{R}^{1}=\mathrm{R}^{2}=\mathrm{Me}(\mathbf{1}), \mathrm{R}^{1}=\mathrm{R}^{2}=\left[-\mathrm{CH}_{2^{-}}\right]_{5}(\mathbf{2})\right\}, \mathrm{Ph}_{2} \mathrm{P}(\mathrm{O}) \mathrm{C}(\mathrm{H})=$ $\mathrm{C}=\mathrm{CR}^{1} \mathrm{R}^{2}\left\{\mathrm{R}^{1}=\mathrm{R}^{2}=\mathrm{Me}(\mathbf{3}), \mathrm{R}^{1}=\mathrm{R}^{2}=\left[-\mathrm{CH}_{2^{-}}\right]_{5}(\mathbf{4})\right\}$ and $(\mathrm{EtO})_{2} \mathrm{P}(\mathrm{O}) \mathrm{C}(\mathrm{H})=\mathrm{C}=\mathrm{CMe}_{2}(\mathbf{5})$ in DMF results in the regiospecific formation of phosphono-acridones and acridonylphosphine oxides. The acridone addition products were also obtained in the reaction of allenes $\mathbf{1}$ and $\mathbf{2}$ with 9-chloroacridine under [Pd]-catalysed conditions, along with (unexpected) $\alpha$-acridinyl substituted allenes. In contrast, 9-benzyl-6-chloro-purine reacted with $\mathbf{1}$ affording a $\beta$-substituted purinone phosphonate. Allenes 1-2 did not react with acridones in the absence of base $(\mathrm{CsF})$, but in the presence of $\mathrm{Pd}(\mathrm{OAc})_{2} / \mathrm{DMF}$ (or DMA)/pivalic acid rearranged to give 1,3-butadienes probably via $[\mathrm{Pd}]$-allyl complexes. The phosphono-acridones were amenable to Horner-Wadsworth-Emmons (HWE) reaction and led to $N$-substituted acridones. Key products have been characterized by single-crystal X-ray crystallography.
\end{abstract}

Keywords. Allenylphosphonate/allenylphosphine oxide; acridone; 9-chloroacridine;

9-benzyl-6-chloro-purine; Horner-Wadsworth-Emmons reaction.

\section{Introduction}

Allenes (or 1,2-dienes) are valuable synthons both industrially and biologically because of the availability of two cumulative double bonds. ${ }^{1,2}$ Allenylphosphonates and allenylphosphine oxides (phosphorylated allenes, e.g., 1-5) that constitute a subclass of allenes are also used as versatile building blocks in organic chemistry. $^{3}$ Our research group has reported that the nucleophilic addition of amines/nucleobases to allenylphosphonates by using $\mathbf{1}$ and related allenes resulted in the formation of $\beta$-aminophosphonates (e.g., 6-8); here stereochemistry of the products depends on the type of amine used. ${ }^{4}$ Recently, rhodium/gold catalysed intermolecular hydroamination of allenes leading to branched allylic amines has been reported independently by Kimber et al., ${ }^{5 \mathrm{a}}$ Breit et al. ${ }^{5 \mathrm{~b}}$ and Widenhoefer et $a l .{ }^{5 c}$ We have previously reported the synthesis of phosphono-benzofurans, ${ }^{6 \mathrm{a}, \mathrm{c}}$ pyrazoles, ${ }^{3 \mathrm{c}}$ -chromenes, ${ }^{6 \mathrm{~b}}$-oxindoles, ${ }^{6 \mathrm{~d}}$ and indolo-lactones ${ }^{6 \mathrm{e}}$ by $[\mathrm{Pd}]$-catalysed reactions from inexpensive phosphorylated allenes. In most of the [Pd]-catalysed reactions using iodoarenes, we observed that the initial

*For correspondence attack occurred at the $\beta$-position of the allenes (cf. scheme 1, compound 9). ${ }^{\text {a }}$ One of our initial objectives was to utilize the analogous reactions of allenes with 9-chloroacridine ${ }^{7}$ to obtain phosphonoacridines, since substituted acridines [e.g., Amsacrine, a drug used in acute lymphoblastic leukemia] are pharmaceutically important. ${ }^{8}$ However, in our reactions using allenylphosphonates, we also obtained acridone (formed by hydrolysis of 9-chloroacridine) addition products. Acridone derivatives are important synthetic targets in medicinal chemistry and pharmaceutical industry and exhibit a variety of biological activities. ${ }^{9 a}$ Recently, Kelly et al. have reported an encouraging results with the use of an acridone derivative against drug-resistant malarial parasites both in vitro and in mice models. ${ }^{9 b, c}$ Studies have also shown that $N$-substituted acridone derivatives display high antiviral activity against the hemorrhagic fever causing arenavirus $\mathrm{JUNV},{ }^{9 \mathrm{~d}}$ bovine viral diarrhea virus (BVDV); ${ }^{9 \mathrm{e}}$ moreover, these derivatives are antagonists to P2X4 receptors and have the ability for the treatment of neuropathic pain and neurodegenerative diseases. ${ }^{9 \mathrm{f}}$ Acridonium-based fluorescent probes were shown to have the ability of detecting the anions (like $\mathrm{F}^{-}, \mathrm{Cl}^{-}, \mathrm{AcO}^{-}$, etc.). ${ }^{9 \mathrm{~g}}$ Apart from these, 

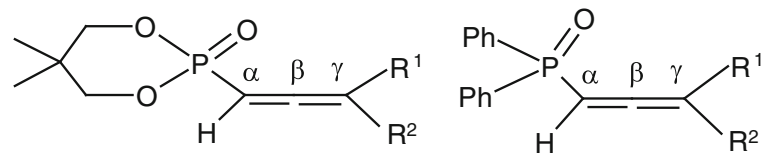

$\mathrm{R}^{1}=\mathrm{R}^{2}=\mathrm{Me} \quad$ (1)

$\mathrm{R}^{1}, \mathrm{R}^{2}=\left[-\mathrm{CH}_{2}-\right]_{5}(\mathbf{2})$

$\mathrm{R}^{1}=\mathrm{R}^{2}=\mathrm{Me} \quad$ (3)

$\mathrm{R}^{1}, \mathrm{R}^{2}=\left[-\mathrm{CH}_{2}-\right]_{5}(4)$

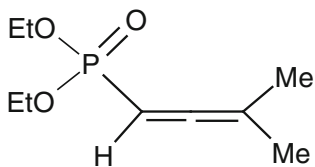

5
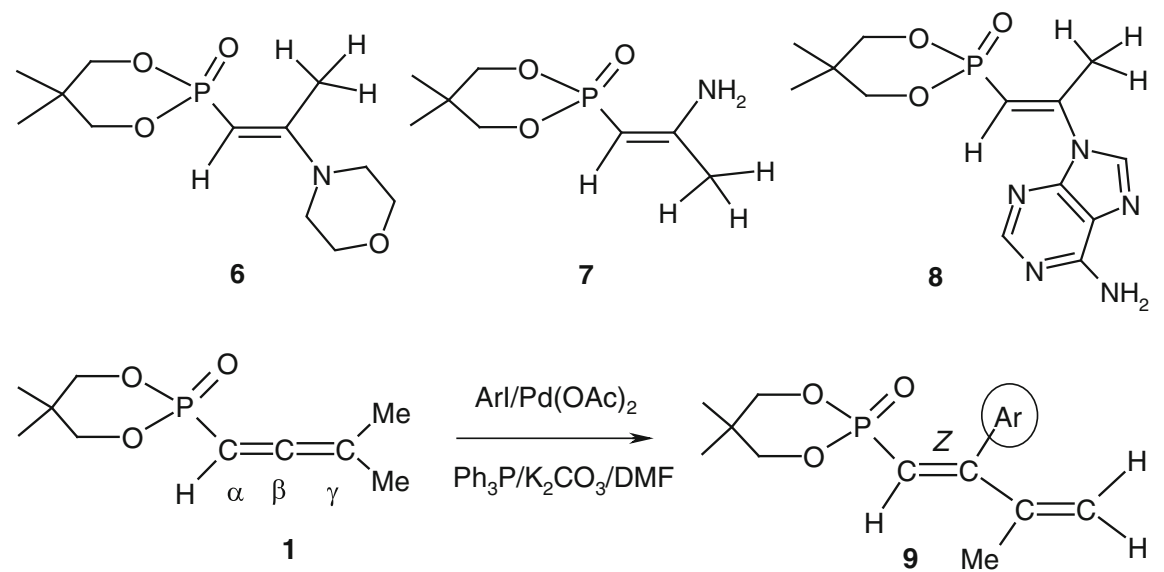

Scheme 1. Palladium catalysed Heck-type arylation showing the attack at the $\beta$-position of allenylphosphonate $\mathbf{1}$.

10 -substituted acridones are used as antipsoriatic agents. ${ }^{9 \mathrm{~h}}$ Therefore, there is continuing interest in the development of an efficient and versatile methods to access $N$-substituted acridone derivatives. Thus, in the first part, we report simple base-mediated addition of $\mathrm{N}-\mathrm{H}$ bond of acridone to phosphorylated allenes 1-5 to give the corresponding phosphonoyl acridone and acridonyl phosphine oxide derivatives in good to high yields. Since these products are obtained alongside the acridinyl allene when [Pd]-catalysed reaction of allenes with 9-chloroacridines, this part will be discussed next. This reaction is then compared with that using 9-benzyl-6-chloropurine. In the presence of the [Pd] catalyst, instead of acridone addition, allene to butadiene rearrangement occurs. This is discussed later. Finally, we have shown that phosphonoyl acridones can be conveniently utilized to prepare $N$-substituted acridones via Horner-Wadsworth-Emmons reaction. ${ }^{10}$

\section{Experimental}

Solvents were dried according to known methods as appropriate. ${ }^{11}{ }^{1} \mathrm{H},{ }^{13} \mathrm{C}$, and ${ }^{31} \mathrm{P}$ NMR spectra $\left({ }^{1} \mathrm{H}\right.$, $400 \mathrm{MHz} ;{ }^{13} \mathrm{C}, 100 \mathrm{MHz} ;{ }^{31} \mathrm{P}, 162 \mathrm{MHz}$ ) were recorded using a $400 \mathrm{MHz}$ spectrometer in $\mathrm{CDCl}_{3}$ with shifts referenced to $\mathrm{SiMe}_{4}(\delta=0)$ or $85 \% \mathrm{H}_{3} \mathrm{PO}_{4}(\delta=0)$. IR spectra were recorded on an FTIR spectrophotometer. Melting points were determined by using a local hot-stage melting point apparatus and are uncorrected.
Elemental analyses were carried out on a CHN analyzer. Mass spectra were recorded using LC-MS and HRMS (ESI-TOF) equipment.

Allenylphosphonates $\mathbf{1 - 3}$ and allenylphosphine oxides 4-5 were prepared by literature methods. ${ }^{12}$ Acridones 10-12 and 9-chloro-acridine were prepared by following a reported method. ${ }^{13}$

\subsection{Representative procedure for the synthesis} of acridonyl allylphosphonates/allylphosphine oxides 13-23

A mixture of allene (one among 1-5) $(1.0 \mathrm{mmol}$ ), acridone (one among 10-12) $(1.0 \mathrm{mmol})$, and $\mathrm{CsF}$ $(0.303 \mathrm{~g}, 2.0 \mathrm{mmol})$ in DMF $(5 \mathrm{~mL})$ was heated at $100^{\circ} \mathrm{C}$ for $4-6 \mathrm{~h}$. When the starting material was consumed fully $\left({ }^{31} \mathrm{P}\right.$ NMR or TLC), the solvent was removed, reaction mixture quenched with water $(5 \mathrm{~mL})$ and extracted with ethyl acetate $(2 \times 30 \mathrm{~mL})$. The combined organic layer was washed with water $(2 \times 20 \mathrm{~mL})$, brine solution $(20 \mathrm{~mL})$, dried $\left(\mathrm{Na}_{2} \mathrm{SO}_{4}\right)$ and then the solvent removed to obtain the crude material which was purified by column chromatography using silica gel with ethyl acetate/hexane (3:2) mixture as eluent to afford the corresponding product among 13-23.

2.1a Compound 13: Yield $0.35 \mathrm{~g}$ (86\%, Pale yellow solid) M.p.: $170-172^{\circ} \mathrm{C}$; IR $\left(\mathrm{KBr}, \mathrm{cm}^{-1}\right)$ 3069, 2957 , 1634, 1601, 1485, 1458, 1264, 1063, 1017. ${ }^{1} \mathrm{H}$ NMR 
$\left(400 \mathrm{MHz}, \mathrm{CDCl}_{3}\right) \delta 0.73$ and $0.91\left(2 \mathrm{~s}, 6 \mathrm{H}, 2 \mathrm{CH}_{3}\right)$, $1.52\left(\mathrm{~d}, 3 \mathrm{H}, J(\mathrm{P}-\mathrm{H})=5.8 \mathrm{~Hz},=\mathrm{CCH}_{3}(\mathrm{~A})\right), 2.24$ $\left(\mathrm{d}, 3 \mathrm{H}, J(\mathrm{P}-\mathrm{H})=4.5 \mathrm{~Hz},=\mathrm{CCH}_{3}(\mathrm{~B})\right), 3.00(\mathrm{~d}, 2 \mathrm{H}$, $\left.J(\mathrm{P}-\mathrm{H})=21.1 \mathrm{~Hz}, \mathrm{PC} H_{2}\right), 3.34(\mathrm{dd} \rightarrow \mathrm{t}, 2 \mathrm{H}, J(\mathrm{P}-\mathrm{H})=$ $\left.J(\mathrm{H}-\mathrm{H}) \sim 11.2 \mathrm{~Hz}, \mathrm{OCH}_{2}\right), 3.78(\mathrm{dd} \rightarrow \mathrm{t}, 2 \mathrm{H}, J(\mathrm{P}-\mathrm{H})=$ $\left.J(\mathrm{H}-\mathrm{H}) \sim 11.6 \mathrm{~Hz}, \mathrm{OCH}_{2}\right), 7.32(\mathrm{t}, 2 \mathrm{H}, J(\mathrm{H}-\mathrm{H})=$ $7.4 \mathrm{~Hz}, \operatorname{Ar}-H), 7.48(\mathrm{~d}, 2 \mathrm{H}, J(\mathrm{H}-\mathrm{H})=8.8 \mathrm{~Hz}, \operatorname{Ar}-H)$, 7.68-7.72 (m, 2H, Ar- $H$ ), 8.56-8.58 (m, 2H, Ar- $H$ ). ${ }^{13} \mathrm{C}$ NMR $\left(100 \mathrm{MHz}, \mathrm{CDCl}_{3}\right) \delta 20.3,20.8,21.3,21.4$, $27.6\left(\mathrm{~d}, J(\mathrm{P}-\mathrm{C})=135.1 \mathrm{~Hz}, \mathrm{PCH}_{2}\right), 32.4(\mathrm{~d}, J(\mathrm{P}-\mathrm{C})=$ $\left.6.0 \mathrm{~Hz}, C(\mathrm{Me})_{2}\right), 75.4,75.5,116.9,119.6,121.9,122.2$, 127.6, 133.8, 140.6, $178.2(C=\mathrm{O}) .{ }^{31} \mathrm{P}$ NMR $(162 \mathrm{MHz}$, $\left.\mathrm{CDCl}_{3}\right) \delta$ 19.5. LC/MS m/z $412[\mathrm{M}+1]^{+}$; Anal.Calcd. for $\mathrm{C}_{23} \mathrm{H}_{26} \mathrm{NO}_{4} \mathrm{P}: \mathrm{C}, 67.14 ; \mathrm{H}, 6.37 ; \mathrm{N}, 3.40$. Found: $\mathrm{C}$, $66.95 ; \mathrm{H}, 6.45 ; \mathrm{N}, 3.56$.

2.1b Compound 14: Yield $0.36 \mathrm{~g}$ (85\%, green solid). M.p.: $172-176^{\circ} \mathrm{C}$; IR $\left(\mathrm{KBr}, \mathrm{cm}^{-1}\right) 3071$, 2973, 1632, 1601, 1495, 1480, 1271, 1059, 1007. ${ }^{1} \mathrm{H}$ NMR $\left(400 \mathrm{MHz}, \mathrm{CDCl}_{3}\right) \quad \delta \quad 0.74$ and $0.94\left(2 \mathrm{~s}, 6 \mathrm{H}, 2 \mathrm{CH}_{3}\right), 1.52(\mathrm{~d}, 3 \mathrm{H}, J(\mathrm{P}-\mathrm{H})=$ $\left.5.6 \mathrm{~Hz},=\mathrm{CCH}_{3}(\mathrm{~A})\right), \quad 2.24(\mathrm{~d}, 3 \mathrm{H}, \quad J(\mathrm{P}-\mathrm{H})=$ $\left.4.4 \mathrm{~Hz},=\mathrm{CCH}_{3}(\mathrm{~B})\right), 2.50\left(\mathrm{~s}, 3 \mathrm{H}, \mathrm{Ar}-\mathrm{CH}_{3}\right), 3.00(\mathrm{~d}, 2 \mathrm{H}$, $\left.J(\mathrm{P}-\mathrm{H})=20.8 \mathrm{~Hz}, \mathrm{PCH}_{2}\right), 3.33-3.38\left(\mathrm{~m}, 2 \mathrm{H}, \mathrm{OCH}_{2}\right)$, 3.35-3.80 (m, 2H, OCH $\left.H_{2}\right), 7.31(\mathrm{t}, 1 \mathrm{H}, J(\mathrm{H}-\mathrm{H})=$ $7.6 \mathrm{~Hz}, \operatorname{Ar}-H), 7.40(\mathrm{~d}, 1 \mathrm{H}, J(\mathrm{H}-\mathrm{H})=8.8 \mathrm{~Hz}, \operatorname{Ar}-H)$, $7.46(\mathrm{~d}, 1 \mathrm{H}, J(\mathrm{H}-\mathrm{H})=8.4 \mathrm{~Hz}, \operatorname{Ar}-H), 7.52-7.55(\mathrm{~m}$, $1 \mathrm{H}, \operatorname{Ar}-H), 7.68-7.71(\mathrm{~m}, 1 \mathrm{H}, \operatorname{Ar}-H), 8.37$ (br s, $1 \mathrm{H}$, Ar- $H), 8.57-8.59$ (m, $1 \mathrm{H}, \mathrm{Ar}-H) .{ }^{13} \mathrm{C}$ NMR $(100 \mathrm{MHz}$, $\left.\mathrm{CDCl}_{3}\right) \delta 20.3\left(\mathrm{~d}, J(\mathrm{P}-\mathrm{C})=3.0 \mathrm{~Hz}, \mathrm{C}=\mathrm{CCH}_{3}\right), 20.7(\mathrm{~d}$, $\left.J(\mathrm{P}-\mathrm{C})=3.0 \mathrm{~Hz}, \mathrm{C}=\mathrm{CCH}_{3}\right), 20.8,21.2,21.4,27.4(\mathrm{~d}$, $\left.J(\mathrm{P}-\mathrm{C})=135.0 \mathrm{~Hz}, \mathrm{PCH}_{2}\right), 32.3(\mathrm{~d}, J(\mathrm{P}-\mathrm{C})=6.0 \mathrm{~Hz}$, $\left.C(\mathrm{Me})_{2}\right), 75.4,75.5,116.8(\mathrm{~d}, J(\mathrm{P}-\mathrm{C})=12.4 \mathrm{~Hz}$, $\left.\mathrm{PCC}=\mathrm{CMe}_{2}\right), 119.7,119.8,121.6,122.0(\mathrm{~d}, J(\mathrm{P}-\mathrm{C})=$ $2.0 \mathrm{~Hz}), 126.7,127.5,131.5,133.5,135.2,138.6,140.4$, 142.1, $142.2,178.0 \quad(C=\mathrm{O}) .{ }^{31} \mathrm{P}$ NMR $(162 \mathrm{MHz}$, $\left.\mathrm{CDCl}_{3}\right) \delta$ 19.5. LC/MS m/z $426[\mathrm{M}]^{+}$. Anal. Calcd. for $\mathrm{C}_{24} \mathrm{H}_{28} \mathrm{NO}_{4} \mathrm{P}: \mathrm{C}, 67.75 ; \mathrm{H}, 6.63 ; \mathrm{N}, 3.29$. Found: $\mathrm{C}$, $67.85 ; \mathrm{H}, 6.54 ; \mathrm{N}, 3.41$.

2.1c Compound 15: Yield $0.39 \mathrm{~g}(80 \%$, yellow solid). M.p.: $160-162^{\circ} \mathrm{C}$; IR $\left(\mathrm{KBr}, \mathrm{cm}^{-1}\right) 2964,2915$, 1633, 1595, 1474, 1458, 1266, 1063, 1019, 811. ${ }^{1} \mathrm{H}$ NMR $\left(400 \mathrm{MHz}, \mathrm{CDCl}_{3}\right) \delta 0.78$ and $0.88(2 \mathrm{~s}$, $\left.6 \mathrm{H}, 2 \mathrm{CH}_{3}\right), 1.51(\mathrm{~d}, 3 \mathrm{H}, J(\mathrm{P}-\mathrm{H})=5.8 \mathrm{~Hz},=$ $\left.\mathrm{CCH}_{3}(\mathrm{~A})\right), \quad 2.23(\mathrm{~d}, \quad 3 \mathrm{H}, \quad J(\mathrm{P}-\mathrm{H})=4.3 \mathrm{~Hz},=$ $\left.\mathrm{CCH}_{3}(\mathrm{~B})\right), 2.92-3.08\left(\mathrm{~m}, 2 \mathrm{H}, \mathrm{PCH}_{2}\right), 3.30-3.47$ (m, $\left.2 \mathrm{H}, \mathrm{OCH}_{2}\right), 3.77-3.87\left(\mathrm{~m}, 2 \mathrm{H}, \mathrm{OCH}_{2}\right), 7.34(\mathrm{t}$, $1 \mathrm{H}, J(\mathrm{H}-\mathrm{H})=7.4 \mathrm{~Hz}, \operatorname{Ar}-H), 7.43(\mathrm{t}, 2 \mathrm{H}, J(\mathrm{H}-$ $\mathrm{H})=8.6 \mathrm{~Hz}, \operatorname{Ar}-H), 7.69-7.76(\mathrm{~m}, 2 \mathrm{H}, \operatorname{Ar}-H)$ $8.56(\mathrm{~d}, 1 \mathrm{H}, J(\mathrm{H}-\mathrm{H})=8.0 \mathrm{~Hz}, \operatorname{Ar}-H), 8.67(\mathrm{~d}, 1 \mathrm{H}$, $J(\mathrm{H}-\mathrm{H})=1.6 \mathrm{~Hz}, \mathrm{Ar}-H) \cdot{ }^{13} \mathrm{C} \mathrm{NMR}\left(100 \mathrm{MHz}, \mathrm{CDCl}_{3}\right)$ $\delta 20.3,20.7,21.3,22.7,25.3,27.6(\mathrm{~d}, J(\mathrm{P}-\mathrm{C})=$ $\left.135.8 \mathrm{~Hz}, \mathrm{PCH}_{2}\right), 32.4\left(\mathrm{~d}, J(\mathrm{P}-\mathrm{C})=5.7 \mathrm{~Hz}, C(\mathrm{Me})_{2}\right)$, $75.3(\mathrm{~d}, J(\mathrm{P}-\mathrm{C})=5.9 \mathrm{~Hz}), 75.5(\mathrm{~d}, J(\mathrm{P}-\mathrm{C})=6.1 \mathrm{~Hz})$, $115.3,116.7,119.2,119.5(\mathrm{~d}, J(\mathrm{P}-\mathrm{C})=10.9 \mathrm{~Hz})$, $122.2(\mathrm{~d}, J(\mathrm{P}-\mathrm{C})=9.2 \mathrm{~Hz}), 123.3,127.7,129.8,134.1$, $136.5,139.4,140.3,142.7(\mathrm{~d}, J(\mathrm{P}-\mathrm{C})=10.2 \mathrm{~Hz}), 177.0$ $(C=\mathrm{O}) ;{ }^{31} \mathrm{P}$ NMR $\left(162 \mathrm{MHz}, \mathrm{CDCl}_{3}\right) \delta 19.6$; HRMS (ESI) Calcd. for $\mathrm{C}_{23} \mathrm{H}_{26} \mathrm{BrNO}_{4} \mathrm{P}\left[\mathrm{M}^{+}+\mathrm{H}\right] 490.0784$ and 492.0784. Found: 490.0783 and 492.0766.

2.1d Compound 16: Yield $0.38 \mathrm{~g}(85 \%$, pale yellow solid). M.p.: $178-180^{\circ} \mathrm{C}$; IR (neat, $\mathrm{cm}^{-1}$ ) 2964, 2926 , 2888, 2241, 1671, 1600, 1375, 1266, 1063, 1014, 734. ${ }^{1} \mathrm{H}$ NMR $\left(400 \mathrm{MHz}, \mathrm{CDCl}_{3}\right) \delta 0.73$ and $0.89(2 \mathrm{~s}, 6 \mathrm{H}$, $\left.2 \mathrm{CH}_{3}\right), 1.41$ (d, 2H, cyclohexyl- $H$ ), 1.59 (br s, $2 \mathrm{H}$, cyclohexyl- $H$ ), 1.86 (br s, 4H, cyclohexyl- $H$ ), 2.66 (br s, $2 \mathrm{H}$, cyclohexyl- $H), 3.01(\mathrm{~d}, 2 \mathrm{H}, J=21.2 \mathrm{~Hz}$, $\left.\mathrm{PCH}_{2}\right), 3.32-3.37\left(\mathrm{~m}, 2 \mathrm{H}, \mathrm{OCH}_{2}\right), 3.75-3.81(\mathrm{~m}, 2 \mathrm{H}$, $\left.\mathrm{OC}_{2}\right), 7.28-7.32(\mathrm{~m}, 2 \mathrm{H}$, Ar-H) $7.52-7.55(\mathrm{~d}, J=$ $8.8 \mathrm{~Hz}, 2 \mathrm{H}, \operatorname{Ar}-H), 7.66-7.70(\mathrm{~m}, 2 \mathrm{H}, \operatorname{Ar}-H), 8.56$ $(\mathrm{d}, J=8.0 \mathrm{~Hz}, 2 \mathrm{H}, \mathrm{Ar}-H) .{ }^{13} \mathrm{C}$ NMR $(100 \mathrm{MHz}$, $\left.\mathrm{CDCl}_{3}\right) \delta 21.3(\mathrm{~d}, J(\mathrm{P}-\mathrm{C})=6.3 \mathrm{~Hz}), 26.0,26.8$, $27.1_{8}, 27.2_{3}\left(\mathrm{~d}, J(\mathrm{P}-\mathrm{C})=134.9 \mathrm{~Hz}, \mathrm{PCH}_{2}\right), 29.9$, $31.1,32.3\left(\mathrm{~d}, J(\mathrm{P}-\mathrm{C})=6.0 \mathrm{~Hz}, C \mathrm{Me}_{2}\right), 75.3(\mathrm{~d}$, $J(\mathrm{P}-\mathrm{C})=6.2 \mathrm{~Hz}), 116.1(\mathrm{~d}, J(\mathrm{P}-\mathrm{C})=10.8 \mathrm{~Hz}), 117.0$, $121.8,122.1,127.4,133.6,140.8,148.8(\mathrm{~d}, J(\mathrm{P}-\mathrm{C})=$ $9.8 \mathrm{~Hz}), 178.1(C=\mathrm{O}) .{ }^{31} \mathrm{P}$ NMR $\left(162 \mathrm{MHz}, \mathrm{CDCl}_{3}\right) \delta$ 19.8. HRMS (ESI) calcd. for $\mathrm{C}_{26} \mathrm{H}_{31} \mathrm{NO}_{4} \mathrm{P}\left[\mathrm{M}^{+}+\mathrm{H}\right]$ : 452.1991. Found: 452.1992.

2.1e Compound 17: Yield $0.41 \mathrm{~g}$ (90\%, brown solid). M.p.: $168-170^{\circ} \mathrm{C}$; IR (KBr, cm $\left.{ }^{-1}\right) 3047$, 2959, 2926, 1638, 1600, 1501, 1479, 1337, 1271, 1063, 1019, 816. ${ }^{1} \mathrm{H}$ NMR $\quad(400 \mathrm{MHz}$, $\left.\mathrm{CDCl}_{3}\right) \delta \quad 0.73$ and $0.90 \quad\left(2 \mathrm{~s}, \quad 6 \mathrm{H}, 2 \mathrm{CH}_{3}\right)$, 1.40-1.41 (m, 2H, cyclohexyl- $H$ ), 1.58 (br s, $2 \mathrm{H}$, cyclohexyl- $H$ ), 1.85 (br s, $4 \mathrm{H}$, cyclohexyl- $H$ ), 2.47 (s, 3H, CH $\left.H_{3}\right), 2.63-2.67(\mathrm{~m}, 2 \mathrm{H}$, cyclohexyl- $H$ ), $3.01\left(\mathrm{~d}, 2 \mathrm{H}, J(\mathrm{P}-\mathrm{H})=20.8 \mathrm{~Hz}, \mathrm{PCH}_{2}\right), 3.35(\mathrm{t}, J=$ $\left.11.0 \mathrm{~Hz}, 2 \mathrm{H}, \mathrm{OCH}_{2}\right), 3.77(\mathrm{~m}, 2 \mathrm{H}, J=11.4 \mathrm{~Hz}, 2 \mathrm{H}$, $\left.\mathrm{OC}_{2}\right), 7.28-7.30(\mathrm{~m}, 1 \mathrm{H}, \mathrm{Ar}-H), 7.44(\mathrm{~d}, J=8.4 \mathrm{~Hz}$, $1 \mathrm{H}, \operatorname{Ar}-H), 7.51(\mathrm{~d}, J=8.4 \mathrm{~Hz}, 2 \mathrm{H}, \operatorname{Ar}-H), 7.65-$ $7.69(\mathrm{~m}, 1 \mathrm{H}, \mathrm{Ar}-\mathrm{H}), 8.34$ (s, $1 \mathrm{H}, \mathrm{Ar}-H), 8.55$ (dd, $J=1.4$ and $8.8 \mathrm{~Hz}, 1 \mathrm{H}, \mathrm{Ar}-H) .{ }^{13} \mathrm{C}$ NMR $(100 \mathrm{MHz}$, $\left.\mathrm{CDCl}_{3}\right) \delta 20.8,21.3(\mathrm{~d}, J(\mathrm{P}-\mathrm{C})=10.1 \mathrm{~Hz}), 26.0,26.8$, $27.1_{5}\left(\mathrm{~d}, J(\mathrm{P}-\mathrm{C})=134.8 \mathrm{~Hz}, \mathrm{PCH}_{2}\right), 27.2,29.9,31.0$, $32.3\left(\mathrm{~d}, J(\mathrm{P}-\mathrm{C})=6.0 \mathrm{~Hz}, C \mathrm{Me}_{2}\right), 75.4(\mathrm{~d}, J(\mathrm{P}-\mathrm{C})=$ $6.0 \mathrm{~Hz}), 116.2(\mathrm{~d}, J(\mathrm{P}-\mathrm{C})=10.8 \mathrm{~Hz}), 116.9\left(\mathrm{~d},{ }^{3} J(\mathrm{P}-\mathrm{C})=\right.$ $12.0 \mathrm{~Hz}), 121.5,122.0,126.7,127.4,131.5,133.4$, $135.1,138.9,140.7,148.7(\mathrm{~d}, J(\mathrm{P}-\mathrm{C})=9.9 \mathrm{~Hz}), 178.0$ $(C=\mathrm{O}) .{ }^{31} \mathrm{P}$ NMR $\left(162 \mathrm{MHz}, \mathrm{CDCl}_{3}\right) \delta$ 19.8. HRMS (ESI) calcd. for $\mathrm{C}_{27} \mathrm{H}_{32} \mathrm{NO}_{4} \mathrm{PNa}\left[\mathrm{M}^{+}+\mathrm{Na}\right] 488.1966$. Found: 488.1961. 
2.1f Compound 18: Yield $0.48 \mathrm{~g}$ (90\%, pale yellow solid). M.p.: $176-178^{\circ} \mathrm{C}$; IR $\left(\mathrm{KBr}, \mathrm{cm}^{-1}\right) 3073,2937$, 2849, 1633, 1600, 1474, 1458, 1255, 1058, 1003, 816. ${ }^{1} \mathrm{H}$ NMR $\left(400 \mathrm{MHz}, \mathrm{CDCl}_{3}\right) \delta 0.79$ and $0.91(2 \mathrm{~s}, 6 \mathrm{H}$, $\left.2 \mathrm{CH}_{3}\right), 1.39-1.43(\mathrm{~m}, 2 \mathrm{H}$, cyclohexyl- $H), 1.58-1.60$ (m, $2 \mathrm{H}$, cyclohexyl- $H$ ), 1.83-1.88 (m, 4H, cyclohexyl$H), 2.64-2.65$ (m, 2H, cyclohexyl- $H$ ), 2.92 (dd, $J=$ 21.2 and $\left.6.3 \mathrm{~Hz}, 1 \mathrm{H}, \mathrm{PC} H_{\mathrm{A}} H_{\mathrm{B}}\right), 3.09(\mathrm{dd}, J=20.9$ and $\left.6.3 \mathrm{~Hz}, 1 \mathrm{H}, \mathrm{PCH}_{\mathrm{A}} H_{\mathrm{B}}\right), 3.32-3.47\left(\mathrm{~m}, 2 \mathrm{H}, \mathrm{OCH}_{2}\right)$, 3.79-3.90 (m, 2H, OC $\left.H_{2}\right), 7.30-7.34(\mathrm{~m}, 1 \mathrm{H}$, Ar- $\mathrm{H})$ $7.49(\mathrm{t}, J=8.6 \mathrm{~Hz}, 2 \mathrm{H}, \mathrm{Ar}-H), 7.68-7.75(\mathrm{~m}, 2 \mathrm{H}, \mathrm{Ar}-$ $H), 8.52-8.54(\mathrm{~m}, 1 \mathrm{H}, \mathrm{Ar}-H), 8.65(\mathrm{~d}, J=2.4 \mathrm{~Hz}, 1 \mathrm{H}$, Ar-H). ${ }^{13} \mathrm{C}$ NMR $\left(100 \mathrm{MHz}, \mathrm{CDCl}_{3}\right) \delta 21.3,25.9,26.7$, 27.1, $27.3\left(\mathrm{~d}, J(\mathrm{P}-\mathrm{C})=135.4 \mathrm{~Hz}, \mathrm{PCH}_{2}\right), 29.9,31.0$, $32.4\left(\mathrm{~d}, J(\mathrm{P}-\mathrm{C})=5.8 \mathrm{~Hz}, C(\mathrm{Me})_{2}\right), 75.2(\mathrm{~d}, J(\mathrm{P}-\mathrm{C})=$ $6.1 \mathrm{~Hz}), 75.4(\mathrm{~d}, J(\mathrm{P}-\mathrm{C})=6.3 \mathrm{~Hz}), 115.2,116.0(\mathrm{~d}$, $J(\mathrm{P}-\mathrm{C})=10.9 \mathrm{~Hz}), 116.9,119.4,122.1,122.2,123.2$, 127.5, 129.6, 134.0, 136.3, 139.7, 140.6, 149.2 (d, $J(\mathrm{P}-\mathrm{C})=10.0 \mathrm{~Hz}), 176.8(C=\mathrm{O}) .{ }^{31} \mathrm{P} \mathrm{NMR}(162 \mathrm{MHz}$, $\left.\mathrm{CDCl}_{3}\right) \delta$ 19.8. HRMS (ESI) calcd. for $\mathrm{C}_{26} \mathrm{H}_{30} \mathrm{BrNO}_{4} \mathrm{P}$ $\left[\mathrm{M}^{+}+\mathrm{H}\right] 530.1097$ and 532.1097. Found: 530.1098 and 532.1085. This compound was crystallized from ethyl acetate/hexane $(1: 1)$ mixture at $25^{\circ} \mathrm{C}$. X-ray structure was determined for this compound.

2.1g Compound 19: Yield 0.39g (85\%, colorless solid). M.p.: $192-194^{\circ} \mathrm{C}$; IR $\left(\mathrm{KBr}, \mathrm{cm}^{-1}\right) 3057,2957$, 2905, 1634, 1601, 1483, 1433, 1265, 1167, $1117 .{ }^{1} \mathrm{H}$ NMR $\left(400 \mathrm{MHz}, \mathrm{CDCl}_{3}\right) \delta 1.52(\mathrm{~d}, 3 \mathrm{H}, J(\mathrm{P}-\mathrm{H})=$ $\left.4.4 \mathrm{~Hz},=\mathrm{CCH}_{3}(\mathrm{~A})\right), 2.24(\mathrm{~d}, 3 \mathrm{H}, J(\mathrm{P}-\mathrm{H})=4.8 \mathrm{~Hz}$, $\left.=\mathrm{CCH}_{3}(\mathrm{~B})\right), 3.60\left(\mathrm{~d}, 2 \mathrm{H}, J(\mathrm{P}-\mathrm{H})=12.8 \mathrm{~Hz}, \mathrm{PCH}_{2}\right)$, 7.10-7.14 (m, 4H, Ar- $H$ ), 7.18-7.22 (m, 2H, Ar- $H$ ), $7.25-7.35(\mathrm{~m}, 6 \mathrm{H}, \operatorname{Ar}-H), 7.43(\mathrm{~d}, J(\mathrm{H}-\mathrm{H})=8.4 \mathrm{~Hz}$, $2 \mathrm{H}$, Ar- $H$ ), 7.56-7.60 (m, 2H, Ar- $H), 8.36-8.39$ (m, $2 \mathrm{H}, \mathrm{Ar}-H) .{ }^{13} \mathrm{C}$ NMR $\left(100 \mathrm{MHz}, \mathrm{CDCl}_{3}\right) \delta 20.5,20.9$, $34.0\left(\mathrm{~d}, J(\mathrm{P}-\mathrm{C})=67.7 \mathrm{~Hz}, \mathrm{PCH}_{2}\right), 117.1,120.9(\mathrm{~d}$, $\left.J(\mathrm{P}-\mathrm{C})=9.1 \mathrm{~Hz}, \mathrm{PCC}=\mathrm{CMe}_{2}\right), 121.5,122.1,127.5$, $128.3,128.4,131.7,132.5(\mathrm{~d}, J(\mathrm{P}-\mathrm{C})=99.5 \mathrm{~Hz}$, $\mathrm{PC}), 133.6,140.3,142.1(\mathrm{~d}, J(\mathrm{P}-\mathrm{C})=8.7 \mathrm{~Hz}), 177.8$ $(C=\mathrm{O}) .{ }^{31} \mathrm{P}$ NMR $\left(162 \mathrm{MHz}, \mathrm{CDCl}_{3}\right) \delta$ 26.4. LC/MS $m / z 464[\mathrm{M}+1]^{+}$.Anal.Calcd. for $\mathrm{C}_{30} \mathrm{H}_{26} \mathrm{NO}_{2} \mathrm{P}: \mathrm{C}$, 77.74; H, 5.65; N, 3.02. Found: C, 77.63; H, 5.58; $\mathrm{N}, 3.12$; This compound was crystallized from ethyl acetate/hexane $(2: 1)$ mixture at $25^{\circ} \mathrm{C}$. X-ray structure was determined for this compound.

2.1h Compound 20: Yield $0.42 \mathrm{~g}$ (88\%, brown solid). M.p.: $110-112^{\circ} \mathrm{C}$; IR $\left(\mathrm{KBr}, \mathrm{cm}^{-1}\right) 3058,2921$, 2855, 1638, 1600, 1496, 1441, 1342, 1293, 1189, 1117, 729. ${ }^{1} \mathrm{H}$ NMR $\left(400 \mathrm{MHz}, \mathrm{CDCl}_{3}\right) \delta 1.48(\mathrm{~d}, 3 \mathrm{H}, J(\mathrm{P}-$ $\left.\mathrm{H})=4.3 \mathrm{~Hz},=\mathrm{CCH}_{3}(\mathrm{~A})\right), 2.31(\mathrm{~d}, 3 \mathrm{H}, J(\mathrm{P}-\mathrm{H})=$ $\left.3.0 \mathrm{~Hz},=\mathrm{CCH}_{3}(\mathrm{~B})\right), 2.42\left(\mathrm{~s}, 3 \mathrm{H}, \mathrm{Ar}-\mathrm{CH}_{3}\right), 3.54(\mathrm{~d}, 2 \mathrm{H}$, $\left.J(\mathrm{P}-\mathrm{H})=12.7 \mathrm{~Hz}, \mathrm{PC} H_{2}\right), 7.06-7.24(\mathrm{~m}, 6 \mathrm{H}, \operatorname{Ar}-H)$,
7.29-7.40 (m, 8H, Ar- $H), 7.53(\mathrm{t}, J=7.1 \mathrm{~Hz}, 1 \mathrm{H}$, Ar- $H$ ), 8.15 (br s, $1 \mathrm{H}, \operatorname{Ar}-H), 8.34(\mathrm{~d}, J=7.9 \mathrm{~Hz}$, $1 \mathrm{H}, \quad$ Ar- $H) .{ }^{13} \mathrm{C}$ NMR $\left(100 \mathrm{MHz}, \mathrm{CDCl}_{3}\right) \delta 20.4$, $20.7\left(\mathrm{~d}, J(\mathrm{P}-\mathrm{C})=15.0 \mathrm{~Hz}, \mathrm{C}=\mathrm{CCH}_{3}\right), 29.7,33.9(\mathrm{~d}$, $J(\mathrm{P}-\mathrm{C})=67.5 \mathrm{~Hz}, \mathrm{PCH} 2), 117.0(\mathrm{~d}, J(\mathrm{P}-\mathrm{C})=5 \mathrm{~Hz}$, $\left.\mathrm{PCC}=\mathrm{CMe}_{2}\right), 120.8(\mathrm{~d}, J(\mathrm{P}-\mathrm{C})=10.6 \mathrm{~Hz}), 121.2$, 121.8, 126.7, 127.4, $128.2(\mathrm{~d}, J(\mathrm{P}-\mathrm{C})=11.5 \mathrm{~Hz})$, 130.0, 131.1, 131.6, $132.5(\mathrm{~d}, J(\mathrm{P}-\mathrm{C})=98.0 \mathrm{~Hz}, \mathrm{PC})$, 132.7, 133.4, 135.0, 138.3, 140.1, $142.0(\mathrm{~d}, J(\mathrm{P}-\mathrm{C})=$ $8.2 \mathrm{~Hz}), 177.6(C=\mathrm{O}) .{ }^{31} \mathrm{P}$ NMR $\left(162 \mathrm{MHz}, \mathrm{CDCl}_{3}\right)$ $\delta$ 26.6. HRMS (ESI) calcd. for $\mathrm{C}_{31} \mathrm{H}_{29} \mathrm{NO}_{2} \mathrm{P}\left[\mathrm{M}^{+}+\mathrm{H}\right]$ 478.1937. Found: 478.1934.

2.1i Compound 21: Yield $0.45 \mathrm{~g}$ (87\%, Yellow solid). M.p.: $140-142^{\circ} \mathrm{C}$; IR $\left(\mathrm{KBr}, \mathrm{cm}^{-1}\right) 3057,2926$, 2854, 1643, 1594, 1495, 1435, 1336, 1292, 1177 , 761. ${ }^{1} \mathrm{H}$ NMR $\left(400 \mathrm{MHz}, \mathrm{CDCl}_{3}\right) \delta 1.39-1.46(\mathrm{~m}$, $2 \mathrm{H}$, cyclohexyl- $H), 1.52-1.60(\mathrm{~m}, 2 \mathrm{H}$, cyclohexyl- $H)$, 1.83-1.87 (m, 4H, cyclohexyl- $H$ ), 2.42 (s, 3H, Ar$\mathrm{CH}_{3}$ ), 2.77 (br s, 2H, cyclohexyl- $H$ ), 3.56 (d, 2H, $\left.J(\mathrm{P}-\mathrm{H})=12.8 \mathrm{~Hz}, \mathrm{PC} H_{2}\right), 7.09-7.16(\mathrm{~m}, 5 \mathrm{H}, \mathrm{Ar}-H)$, 7.19-7.36 (m, 7H, Ar- $H$ ), 7.43-7.54 (m, 3H, Ar- H), $8.16(\mathrm{~s}, 1 \mathrm{H}, \operatorname{Ar}-H), 8.34(\mathrm{~d}, J=8.0 \mathrm{~Hz}, 1 \mathrm{H}, \operatorname{Ar}-H)$. ${ }^{13} \mathrm{C}$ NMR $\left(100 \mathrm{MHz}, \mathrm{CDCl}_{3}\right) \delta 14.1,20.7,22.7,26.1$, 27.0, 27.6, 29.7, 30.1, 31.2, 32.0, $33.3(\mathrm{~d}, J(\mathrm{P}-\mathrm{C})=$ $\left.67.6 \mathrm{~Hz}, \mathrm{PCH}_{2}\right), 36.7,117.1,121.2,121.8,126.6$, $127.3,128.3\left(\mathrm{~d}, J(\mathrm{P}-\mathrm{C})=11.5 \mathrm{~Hz}, \mathrm{PCC}=\mathrm{CMe}_{2}\right)$, 130.0, 131.1, $132.3(\mathrm{~d}, J(\mathrm{P}-\mathrm{C})=98.5 \mathrm{~Hz}, \mathrm{PC}), 132.6$, 133.3, 135.0, 138.7, 140.5, 149.0, $177.7(C=\mathrm{O}) .{ }^{31} \mathrm{P}$ NMR $\left(162 \mathrm{MHz}, \mathrm{CDCl}_{3}\right) \delta$ 26.8. HRMS (ESI) calcd. for $\mathrm{C}_{34} \mathrm{H}_{33} \mathrm{NO}_{2} \mathrm{P}\left[\mathrm{M}^{+}+\mathrm{H}\right]$ 518.2249. Found: 518.2247.

2.1j Compound 22: Yield $0.52 \mathrm{~g}$ (90\%, pale yellow solid). M.p.: $194-196^{\circ} \mathrm{C}$; IR $\left(\mathrm{KBr}, \mathrm{cm}^{-1}\right) 3057$, 2970, 2926, 2854, 1638, 1479, 1332, 1271, 1167, 1123, 750. ${ }^{1} \mathrm{H}$ NMR $\left(400 \mathrm{MHz}, \mathrm{CDCl}_{3}\right) \delta 1.35-1.46$ $(\mathrm{m}, 2 \mathrm{H}$, cyclohexyl- $H), 1.60-1.62(\mathrm{~m}, 2 \mathrm{H}$, cyclohexyl$H$ ), 1.83-1.93 (m, 4H, cyclohexyl- $H$ ), 2.76 (br s, $2 \mathrm{H}$, cyclohexyl- $H$ ), 3.44 (dd, $J=20.1$ and $3.8 \mathrm{~Hz}$, $\left.1 \mathrm{H}, \mathrm{PC} H_{\mathrm{A}} H_{\mathrm{B}}\right), 3.63(\mathrm{dd}, J=22.1$ and $3.8 \mathrm{~Hz}, 1 \mathrm{H}$, $\left.\mathrm{PCH}_{\mathrm{A}} H_{\mathrm{B}}\right), 7.06-7.10(\mathrm{~m}, 2 \mathrm{H}, \operatorname{Ar}-H), 7.16-7.20(\mathrm{~m}$, $2 \mathrm{H}, \operatorname{Ar}-H), 7.23-7.32(\mathrm{~m}, 5 \mathrm{H}, \operatorname{Ar}-H), 7.36-7.46$ $(\mathrm{m}, 4 \mathrm{H}, \operatorname{Ar}-H), 7.52-7.55(\mathrm{~m}, 1 \mathrm{H}$, Ar- $H), 7.60-7.63$ $(\mathrm{m}, 1 \mathrm{H}, \operatorname{Ar}-H), 8.37-8.40(\mathrm{~m}, 2 \mathrm{H}, \mathrm{Ar}-H) .{ }^{13} \mathrm{C}$ NMR $\left(100 \mathrm{MHz}, \mathrm{CDCl}_{3}\right) \delta 26.0,26.9,27.6,30.1,31.3$, $33.3\left(\mathrm{~d}, J(\mathrm{P}-\mathrm{C})=67.4 \mathrm{~Hz}, \mathrm{PCH}_{2}\right), 114.9,117.0$, $120.0,122.1\left(\mathrm{~d}, J(\mathrm{P}-\mathrm{C})=15.6 \mathrm{~Hz}, \mathrm{PCC}=\mathrm{CMe}_{2}\right)$, 123.0, 127.6, 128.2, 128.3, 128.4, 128.5, 129.5, 130.0, $131.7,131.8(\mathrm{~d}, J(\mathrm{P}-\mathrm{C})=96.8 \mathrm{~Hz}, \mathrm{PC}), 132.5,133.4$, 133.9, 136.0, 139.5, 140.4, 149.4, $176.5(C=\mathrm{O}) .{ }^{31} \mathrm{P}$ NMR $\left(162 \mathrm{MHz}, \mathrm{CDCl}_{3}\right) \delta$ 25.9. HRMS (ESI) calcd. for $\mathrm{C}_{33} \mathrm{H}_{30} \mathrm{BrNO}_{2} \mathrm{P}\left[\mathrm{M}^{+}+\mathrm{H}\right] 582.1198$ and 584.1198. Found: 582.1193 and 584.1176. 
2.1k Compound 23: Yield $0.35 \mathrm{~g}$ (88\%, gummy liquid); IR (neat, $\mathrm{cm}^{-1}$ ) 3069, 2975, 2915, 1638, $1605,1479,1458,1353,1260,1030,767 .{ }^{1} \mathrm{H}$ NMR $\left(400 \mathrm{MHz}, \mathrm{CDCl}_{3}\right) \delta 0.90\left(\mathrm{t}, J=7.0 \mathrm{~Hz}, 6 \mathrm{H}, 2 \mathrm{CH}_{3}\right)$, $1.50\left(\mathrm{~d}, 3 \mathrm{H}, J(\mathrm{P}-\mathrm{H})=5.6 \mathrm{~Hz},=\mathrm{CCH}_{3}(\mathrm{~A})\right), 2.17(\mathrm{~d}$, $\left.3 \mathrm{H}, J(\mathrm{P}-\mathrm{H})=4.4 \mathrm{~Hz},=\mathrm{CCH}_{3}(\mathrm{~B})\right), 2.96(\mathrm{~d}, 2 \mathrm{H}, J(\mathrm{P}-$ $\left.\mathrm{H})=22.0 \mathrm{~Hz}, \mathrm{PC} \mathrm{H}_{2}\right), 3.67-3.79\left(\mathrm{~m}, 4 \mathrm{H}, 2 \mathrm{OCH}_{2}\right)$, 7.27-7.31 (m, 2H, Ar- $H), 7.42(\mathrm{~d}, 2 \mathrm{H}, J=8.4 \mathrm{~Hz}$, Ar$H$ ), 7.65-7.69 (m, 2H, Ar- $H$ ), 8.54 (d, $2 \mathrm{H}, J=1.2 \mathrm{~Hz}$, $\mathrm{Ar}-H) .{ }^{13} \mathrm{C}$ NMR $\left(100 \mathrm{MHz}, \mathrm{CDCl}_{3}\right) \delta 15.9(\mathrm{~d}, J(\mathrm{P}-$ $\left.\mathrm{C})=5.8 \mathrm{~Hz}, \mathrm{OCH}_{2} \mathrm{CH}_{3}\right), 20.4(\mathrm{~d}, J(\mathrm{P}-\mathrm{C})=21.4 \mathrm{~Hz}$, $\left.\mathrm{C}=\mathrm{CCH}_{3}\right), 29.1\left(\mathrm{~d}, J(\mathrm{P}-\mathrm{C})=141.0 \mathrm{~Hz}, \mathrm{PCH}_{2}\right), 61.7$ $\left(\mathrm{d}, J(\mathrm{P}-\mathrm{C})=6.2 \mathrm{~Hz}, \mathrm{OCH}_{2} \mathrm{CH}_{3}\right), 120.7(\mathrm{~d}, J(\mathrm{P}-\mathrm{C})=$ $\left.12.2 \mathrm{~Hz}, \mathrm{PCC}=\mathrm{CMe}_{2}\right), 121.7,122.1,127.5,133.5$, 140.7, $141.0(\mathrm{~d}, J(\mathrm{P}-\mathrm{C})=10.5 \mathrm{~Hz}), 178.1(C=\mathrm{O}) .{ }^{31} \mathrm{P}$ NMR $\left(162 \mathrm{MHz}, \mathrm{CDCl}_{3}\right) \delta$ 24.2. HRMS (ESI) calcd. for $\mathrm{C}_{22} \mathrm{H}_{27} \mathrm{NO}_{4} \mathrm{P}\left[\mathrm{M}^{+}+\mathrm{H}\right]$ 400.1678. Found: 400.1680 .

\subsection{Synthesis of $\alpha$-acridinyl allenylphosphonate derivatives 24 and 25}

A mixture of allene (1 or 2) $(0.216 \mathrm{~g}, 1.0 \mathrm{mmol})$, 9chloroacridine $(0.213 \mathrm{~g}, 1.0 \mathrm{mmol}), \mathrm{Pd}(\mathrm{OAc})_{2}(5 \mathrm{~mol} \%)$ and $\mathrm{CsF}(0.303 \mathrm{~g}, 2.0 \mathrm{mmol})$ in DMF $(5 \mathrm{~mL})$ was heated at $100^{\circ} \mathrm{C}$ for $4-6 \mathrm{~h}$. When the starting material was consumed fully $\left({ }^{31} \mathrm{P}\right.$ NMR or TLC), the solvent was removed, reaction mixture quenched with water $(5 \mathrm{~mL})$ and extracted with ethyl acetate $(2 \times 30 \mathrm{~mL})$. The combined organic layer was washed with water $(2 \times 20 \mathrm{~mL})$, brine solution $(20 \mathrm{~mL})$, dried $\left(\mathrm{Na}_{2} \mathrm{SO}_{4}\right)$ and then the solvent was removed to obtain the crude material which was purified by column chromatography using silica gel with ethyl acetate/hexane (3:2) mixture as eluent to afford the corresponding product $\mathbf{2 4}$ or $\mathbf{2 5}$. Reaction using $\mathrm{PPh}_{3} / \mathrm{K}_{2} \mathrm{CO}_{3}$ in place of $\mathrm{CsF}$ did not give these products. Note: Along with these, we obtained the corresponding acridone derivatives also. Rest of the allenes (3-5), when treated with 9-chloroacridine gave only acridone derivatives.

2.2a Compound 24: Yield 0.08 g [20\%, white solid; combined yield of $\mathbf{1 3}+\mathbf{2 4}$ was $>90 \%]$. Melting point (M.p.): $184-186^{\circ} \mathrm{C}$; IR $\left(\mathrm{KBr}, \mathrm{cm}^{-1}\right) 2946,2907$, 1958, 1634, 1601, 1485, 1458, 1265, 1061, 1011. ${ }^{1} \mathrm{H}$ NMR $\left(400 \mathrm{MHz}, \mathrm{CDCl}_{3}\right) \delta 0.47$ and $0.88(2 \mathrm{~s}$, $\left.6 \mathrm{H}, 2 \mathrm{CH}_{3}\right), 1.91-1.93\left(\mathrm{~m}, 6 \mathrm{H},=\mathrm{C}\left(\mathrm{CH}_{3}\right)_{2}\right), 3.57-$ $3.61\left(\mathrm{~m}, 2 \mathrm{H}, \mathrm{OCH}_{2}\right), 4.12-4.18\left(\mathrm{~m}, 2 \mathrm{H}, \mathrm{OCH}_{2}\right)$, $7.61(\mathrm{~d}, 2 \mathrm{H}, J(\mathrm{H}-\mathrm{H}) \sim 7.8 \mathrm{~Hz}, \operatorname{Ar}-H), 7.82(\mathrm{~d}, 2 \mathrm{H}$, $J(\mathrm{H}-\mathrm{H}) \sim 7.8 \mathrm{~Hz}$, Ar- $H), 8.27(\sim \mathrm{t}, 2 \mathrm{H}, J(\mathrm{H}-\mathrm{H})$ $\sim 7.8 \mathrm{~Hz}, \operatorname{Ar}-H), 8.38(\sim \mathrm{t}, 2 \mathrm{H}, J(\mathrm{H}-\mathrm{H}) \sim 7.8 \mathrm{~Hz}, \mathrm{Ar}-$ $H) .{ }^{13} \mathrm{C}$ NMR $\left(100 \mathrm{MHz}, \mathrm{CDCl}_{3}\right) \delta 19.0,19.1,20.8$, 21.3, $32.2\left(\mathrm{~d}, J(\mathrm{P}-\mathrm{C})=6.0 \mathrm{~Hz}, C \mathrm{Me}_{2}\right), 75.7,75.8$, $88.8(\mathrm{~d}, J(\mathrm{P}-\mathrm{C})=199.1 \mathrm{~Hz}, \mathrm{P}-C=\mathrm{C}=\mathrm{C}), 99.3(\mathrm{~d}, J(\mathrm{P}-$ C) $=16.0 \mathrm{~Hz}, \mathrm{P}-\mathrm{C}=\mathrm{C}=C), 125.2,125.3,126.0,126.5$, 129.8, 130.1, 138.5, 148.9, 211.4. ${ }^{31} \mathrm{P}$ NMR $(162 \mathrm{MHz}$, $\left.\mathrm{CDCl}_{3}\right) \delta$ 8.7. LC/MS $m / z 285[\mathrm{M}+1]^{+}$; Anal.Calcd. for $\mathrm{C}_{23} \mathrm{H}_{24} \mathrm{NO}_{3} \mathrm{P}: \mathrm{C}, 70.22 ; \mathrm{H}, 6.15 ; \mathrm{N}, 3.56$. Found: $\mathrm{C}$, $70.12 ; \mathrm{H}, 6.10 ; \mathrm{N}, 3.61$. This compound was crystallized from ethyl acetate/hexane $(1: 1)$ mixture at $25^{\circ} \mathrm{C}$. $\mathrm{X}$-ray structure was determined for this compound.

2.2b Compound 25: Yield 0.09g (20\%, pale brown solid; combined yield of $\mathbf{1 6}+\mathbf{2 5}$ by ${ }^{31} \mathrm{P}$ NMR was >95\%). M.p.: $210-212^{\circ} \mathrm{C}$; IR $\left(\mathrm{KBr}, \mathrm{cm}^{-1}\right)$ 2975, 2926, 2849, 1967, 1539, 1457, 1325, 1260, 1057, 1002, 986, 838, 777. ${ }^{1} \mathrm{H}$ NMR $\left(400 \mathrm{MHz}, \mathrm{CDCl}_{3}\right) \delta 0.52$ and $0.90\left(2 \mathrm{~s}, 6 \mathrm{H}, 2 \mathrm{CH}_{3}\right), 1.42-1.47$ (m, 4H, cyclohexyl$H$ ), 1.71-1.73 (m, 2H, cyclohexyl- $H$ ), 2.24-2.29 (m, $2 \mathrm{H}$, cyclohexyl- $H), 2.43-2.48(\mathrm{~m}, 2 \mathrm{H}$, cyclohexyl- $H)$, 3.60-3.66 (m, 2H, OC $\left.H_{2}\right), 4.06-4.11\left(\mathrm{~m}, 2 \mathrm{H}, \mathrm{OCH}_{2}\right)$, $7.58(\mathrm{t}, J=7.6 \mathrm{~Hz}, 2 \mathrm{H}, \operatorname{Ar}-H), 7.79(\mathrm{t}, J=7.6 \mathrm{~Hz}$, $2 \mathrm{H}, \operatorname{Ar}-H), 8.24(\mathrm{~d}, 2 \mathrm{H}, J=8.8 \mathrm{~Hz}, \operatorname{Ar}-H), 8.36$ $(\mathrm{d}, J=8.8 \mathrm{~Hz}, 2 \mathrm{H}, \mathrm{Ar}-\mathrm{H}) \cdot{ }^{13} \mathrm{C} \mathrm{NMR}(100 \mathrm{MHz}$, $\left.\mathrm{CDCl}_{3}\right) \delta 20.9,21.3,25.6,26.5,29.7,32.3(\mathrm{~d}, J(\mathrm{P}-$ C) $\left.=6.0 \mathrm{~Hz}, C \mathrm{Me}_{2}\right), 75.9(\mathrm{~d}, J(\mathrm{P}-\mathrm{C})=6.0 \mathrm{~Hz}, \mathrm{P}-$ $\mathrm{O}-C), 88.2(\mathrm{~d}, J(\mathrm{P}-\mathrm{C})=197.0 \mathrm{~Hz}, \mathrm{P}-C), 105.6(\mathrm{~d}$, $J(\mathrm{P}-\mathrm{C})=15.0 \mathrm{~Hz}, \mathrm{P}-\mathrm{C}=\mathrm{C}=C), 125.3,126.0,126.6$, 129.6, 130.1, 138.8, 148.8, 208.1. ${ }^{31} \mathrm{P}$ NMR (162 MHz, $\left.\mathrm{CDCl}_{3}\right) \delta$ 8.9. HRMS (ESI) calcd. for $\mathrm{C}_{26} \mathrm{H}_{29} \mathrm{NO}_{3} \mathrm{P}$ $[\mathrm{M}+\mathrm{H}]^{+}:$434.1886. Found: 434.1882. This compound was crystallized from ethyl acetate/hexane (1:1) mixture at $25^{\circ} \mathrm{C}$. X-ray structure was determined for this compound.

\subsection{Synthesis of phosphono-purine derivative $\mathbf{2 7}$}

In a $25 \mathrm{~mL}$ round-bottomed flask, a mixture of allene (0.098 g, $0.4 \mathrm{mmol})$, 9-benzyl 6-chloro-purine (0.100 g, $0.4 \mathrm{mmol}), \mathrm{Pd}(\mathrm{OAc})_{2}(0.092 \mathrm{~g}, 0.04 \mathrm{mmol})$ and $\mathrm{K}_{2} \mathrm{CO}_{3}$ $(0.113 \mathrm{~g}, 0.81 \mathrm{mmol}), \mathrm{PPh}_{3}(0.032 \mathrm{~g}, 0.12 \mathrm{mmol})$ in DMF $(2 \mathrm{~mL})$ solvent was heated at $100^{\circ} \mathrm{C}$ for $12 \mathrm{~h}$. When the starting material was consumed fully $\left({ }^{31} \mathrm{P}\right.$ NMR or TLC), the solvent was removed, reaction mixture quenched with water $(5 \mathrm{~mL})$ and extracted with ethyl acetate $(2 \times 20 \mathrm{~mL})$. The combined organic layer was washed with water $(2 \times 10 \mathrm{~mL})$, brine solution $(10 \mathrm{~mL})$, dried $\left(\mathrm{Na}_{2} \mathrm{SO}_{4}\right)$ and then the solvent was removed to obtain the crude material which was purified by column chromatography using silica gel with ethyl acetate/methanol (9:1) mixture as eluent to afford the product 27 . Yield $0.127 \mathrm{~g}(67 \%$, white solid). M.p.: 228-230 ${ }^{\circ}$; IR (neat, $\mathrm{cm}^{-1}$ ) 3112, 3063, 3030, 2948, 2849, 1677, 1573, 1540, 1507, 1441, 1358, 1266, 1057, 1008, 816. ${ }^{1} \mathrm{H}$ NMR $\left.\left(400 \mathrm{MHz}, \mathrm{CDCl}_{3}\right)\right) \delta 1.00(\mathrm{~s}, 6 \mathrm{H}$, 
$\left.2 \mathrm{CH}_{3}\right), 1.48-1.60$ (m, 4H, cyclohexyl- $\left.H\right), 1.74-1.75$ (m, 2H, cyclohexyl- $H$ ), 1.90-1.97 (m, 2H, cyclohexyl$H), 2.31-2.47$ (m, 2H, cyclohexyl- $H), 3.14(\mathrm{dd}, 1 \mathrm{H}$, $J=16.4$ and $\left.3.2 \mathrm{~Hz}, \mathrm{PCH}_{\mathrm{A}} \mathrm{H}_{\mathrm{B}}\right), 3.37(\mathrm{dd}, J=16.0$ and $\left.3.2 \mathrm{~Hz}, 1 \mathrm{H}, \mathrm{PCH}_{\mathrm{A}} H_{\mathrm{B}}\right), 3.64(\mathrm{t}, J=11.8 \mathrm{~Hz}, 1 \mathrm{H}$, $\left.\mathrm{OC}_{\mathrm{A}} \mathrm{H}_{\mathrm{B}}\right), 3.83\left(\mathrm{t}, J=11.8 \mathrm{~Hz}, 1 \mathrm{H}, \mathrm{OCH}_{\mathrm{A}} H_{\mathrm{B}}\right), 4.00(\mathrm{t}$, $\left.J=10.8 \mathrm{~Hz}, 1 \mathrm{H}, \mathrm{OC}_{\mathrm{A}} \mathrm{H}_{\mathrm{B}}\right), 4.14(\mathrm{t}, J=10.8 \mathrm{~Hz}, 1 \mathrm{H}$, $\left.\mathrm{OCH}_{\mathrm{A}} H_{\mathrm{B}}\right), 5.32\left(\mathrm{~s}, 2 \mathrm{H}, \mathrm{CH}_{2} \mathrm{Ar}\right), 7.30-7.38(\mathrm{~m}, 5 \mathrm{H}, \mathrm{Ar}-$ $\mathrm{H}), 7.72(\mathrm{~s}, 1 \mathrm{H}, \operatorname{Ar}-H), 7.80(\mathrm{~s}, 1 \mathrm{H}, \operatorname{Ar}-H) .{ }^{13} \mathrm{C} \mathrm{NMR}$ $\left(100 \mathrm{MHz}, \mathrm{CDCl}_{3}\right) \delta 21.4(\mathrm{~d}, J(\mathrm{P}-\mathrm{C})=11.0 \mathrm{~Hz})$, 26.0, 26.2, 27.1, 27.5, $30.4(\mathrm{~d}, J(\mathrm{P}-\mathrm{C})=94.0 \mathrm{~Hz})$, $32.6\left(\mathrm{~d}, J(\mathrm{P}-\mathrm{C})=5.8 \mathrm{~Hz}, C \mathrm{Me}_{2}\right), 47.6,75.3(\mathrm{~d}, J(\mathrm{P}-$ $\mathrm{C})=6.2 \mathrm{~Hz}), 75.6(\mathrm{~d}, J(\mathrm{P}-\mathrm{C})=6.2 \mathrm{~Hz}), 118.9(\mathrm{~d}$, $J(\mathrm{P}-\mathrm{C})=12.5 \mathrm{~Hz}), 124.0,128.0,128.6,129.1,135.0$, $140.0,144.7(\mathrm{~d}, J(\mathrm{P}-\mathrm{C})=11.1 \mathrm{~Hz}), 148.0,149.3$, 156.6. ${ }^{31} \mathrm{P}$ NMR $\left(162 \mathrm{MHz}, \mathrm{CDCl}_{3}\right) \delta$ 21.6. HRMS (ESI) calcd. for $\mathrm{C}_{25} \mathrm{H}_{31} \mathrm{~N}_{4} \mathrm{O}_{4} \mathrm{PNa}\left[\mathrm{M}^{+}+\mathrm{Na}\right]$ : 505.1981. Found: 505.2004. X-ray structure was determined for this compound after crystallization from methanol.

\subsection{Synthesis of phosphono-butadienes $\mathbf{2 8}$ and $\mathbf{2 9}$} by using allenylphosphonates $\mathbf{1}$ and $\mathbf{2}$

To $\mathrm{Pd}(\mathrm{OAc})_{2} \quad(0.14 \mathrm{mmol})$ and allenylphosphonate (1 or 2) $(1.4 \mathrm{mmol})$ in a $25 \mathrm{~mL} \mathrm{RBF}$, was added dimethylformamide (DMF) [or dimethylacetamide (DMA)]/ pivalic acid mixture $(5 \mathrm{~mL} ; 4: 1)$ and then the reaction mixture was gradually heated from room temperature to $120^{\circ} \mathrm{C}$ in air with the stirring continued for $1 \mathrm{~h}$. The mixture was cooled to $25^{\circ} \mathrm{C}$, quenched with water $(5 \mathrm{~mL})$ and extracted with EtOAc $(3 \times 20 \mathrm{~mL})$. The whole organic layer was washed with water $(3 \times$ $20 \mathrm{~mL})$, dried $\left(\mathrm{Na}_{2} \mathrm{SO}_{4}\right)$, filtered, and filtrate concentrated. The residue was subjected to column chromatography (hexane/EtOAc; 3:2) to afford the product 28 or 29.

2.4a Compound 28: Yield $0.124 \mathrm{~g}$ (62\% white solid). M.p.: $90-92^{\circ} \mathrm{C}$; IR ( $\left.\mathrm{KBr}, \mathrm{cm}^{-1}\right)$ 3084, 3002, 2964, 2876, 1632, 1595, 1479, 1375, 1255, 1058, 1003, 866. ${ }^{1} \mathrm{H}$ NMR $\left(400 \mathrm{MHz}, \mathrm{CDCl}_{3}\right) \delta 1.04(\mathrm{~s}, 3 \mathrm{H}, \mathrm{CH})$, $1.11\left(\mathrm{~s}, 3 \mathrm{H}, \mathrm{CH}_{3}\right), 1.87\left(\mathrm{~s}, 3 \mathrm{H}, \mathrm{CH}_{3}\right), 3.80-3.87(\mathrm{~m}$, $\left.2 \mathrm{H}, \mathrm{OCH}_{2}\right), 4.17-4.23\left(\mathrm{~m}, 2 \mathrm{H}, \mathrm{OCH}_{2}\right), 5.34-5.36(\mathrm{~m}$, $\left.2 \mathrm{H},=\mathrm{CH}_{2}\right), 5.66-5.75(\mathrm{dd} \rightarrow \mathrm{t}, J \sim 18.0 \mathrm{~Hz}, 1 \mathrm{H},=$ $\mathrm{CH}), 7.23-7.27(\mathrm{~m}, 1 \mathrm{H},=\mathrm{CH}) .{ }^{13} \mathrm{C}$ NMR $(100 \mathrm{MHz}$, $\left.\mathrm{CDCl}_{3}\right) \delta 17.7,21.4,21.6,32.5(\mathrm{~d}, J(\mathrm{PC})=5.7 \mathrm{~Hz})$, $75.4(\mathrm{~d}, J(\mathrm{PC})=5.8 \mathrm{~Hz}), 112.0(\mathrm{~d}, J(\mathrm{PC})=190.1 \mathrm{~Hz})$, $124.7,140.7(\mathrm{~d}, J(\mathrm{PC})=23.9 \mathrm{~Hz}), 152.9(\mathrm{~d}, J(\mathrm{PC})=$ $5.9 \mathrm{~Hz}) .{ }^{31} \mathrm{P}$ NMR $\left(162 \mathrm{MHz}, \mathrm{CDCl}_{3}\right) \delta$ 15.4. HRMS (ESI) calcd. for $\mathrm{C}_{10} \mathrm{H}_{18} \mathrm{O}_{3} \mathrm{P}\left[\mathrm{M}^{+}+\mathrm{H}\right]:$ 217.0993. Found:
217.0993. This compound had been previously prepared in our laboratory; the IR/NMR data are consistent with the ones reported before. ${ }^{14}$

2.4b Compound 29: Yield $0.136 \mathrm{~g}$ (67\% white solid). M.p.: $120-122^{\circ} \mathrm{C}$; IR $\left(\mathrm{KBr}, \mathrm{cm}^{-1}\right)$ 3036, 3013, 2926, 2893, 1627, 1589, 1474, 1260, 1052, 1014, 860. ${ }^{1} \mathrm{H}$ NMR $\left(400 \mathrm{MHz}, \mathrm{CDCl}_{3}\right) \delta 1.02\left(\mathrm{~s}, 3 \mathrm{H}, \mathrm{CH}_{3}\right)$, $1.11\left(\mathrm{~s}, 3 \mathrm{H}, \mathrm{CH}_{3}\right), 1.59-1.70(\mathrm{~m}, 4 \mathrm{H}$, cyclohexenyl$H$ ), 2.12-2.20 (m, 4H, cyclohexenyl- $H$ ), 3.78-3.84 (m, $\left.2 \mathrm{H}, \mathrm{OCH}_{2}\right), 4.20\left(\mathrm{dd} \rightarrow \mathrm{t}, J=10.2 \mathrm{~Hz}, 2 \mathrm{H}, \mathrm{OCH}_{2}\right)$, $5.55(\mathrm{dd} \rightarrow \mathrm{t}, J \sim 18.0 \mathrm{~Hz}, 1 \mathrm{H},=\mathrm{CH}), 6.15(\mathrm{br} \mathrm{s}$, $1 \mathrm{H},=\mathrm{CH}), 7.10-7.20(\mathrm{dd}, J \sim 18.0,22.8 \mathrm{~Hz}, 1 \mathrm{H},=$ $\mathrm{CH}) .{ }^{13} \mathrm{C}$ NMR $\left(100 \mathrm{MHz}, \mathrm{CDCl}_{3}\right) \delta 21.4,21.6,22.0$, $23.7,26.3,32.4(\mathrm{~d}, J(\mathrm{PC})=5.5 \mathrm{~Hz}), 75.2(\mathrm{~d}, J(\mathrm{PC})=$ $5.8 \mathrm{~Hz}), 107.2(\mathrm{~d}, J(\mathrm{PC})=192.0 \mathrm{~Hz}), 135.2(\mathrm{~d}, J(\mathrm{PC})$ $=23.9 \mathrm{~Hz}), 139.2,153.6(\mathrm{~d}, J(\mathrm{PC})=6.2 \mathrm{~Hz}) .{ }^{31} \mathrm{P}$ NMR $\left(162 \mathrm{MHz}, \mathrm{CDCl}_{3}\right) \delta$ 17.1. HRMS (ESI) calcd. for $\mathrm{C}_{13} \mathrm{H}_{22} \mathrm{O}_{3} \mathrm{P}\left[\mathrm{M}^{+}+\mathrm{H}\right]: 257.1306$. Found: 257.1306.

\subsection{Horner-Wadsworth-Emmons reaction using the acridonyl allylphosphonate 16: Synthesis of alkenyl-acridone derivatives 30-37}

The phosphonate $16(0.100 \mathrm{~g}, 0.22 \mathrm{mmol})$ was dissolved in dry THF ( $2 \mathrm{~mL})$ and added drop-wise (10 min) to a suspension of $\mathrm{NaH}(0.44 \mathrm{mmol})$ in THF $(3 \mathrm{~mL})$ at $0^{\circ} \mathrm{C}$ with stirring. The mixture was stirred further at this temperature for $0.5 \mathrm{~h}$. Then aldehyde $(0.33 \mathrm{mmol})$ in THF $(2 \mathrm{~mL})$ was added and the mixture stirred for $12 \mathrm{~h}$ at room temperature. Water $(10 \mathrm{~mL})$ was added and the aqueous layer thoroughly extracted with ethyl acetate $(3 \times 20 \mathrm{~mL})$. The organic layer was collected, dried $\left(\mathrm{Na}_{2} \mathrm{SO}_{4}\right)$, filtered and the solvent removed from the filtrate to give a residue that was purified by column chromatography [silica gel, ethyl acetate-hexane (1:4)] to give one of the compounds $\mathbf{3 0}-\mathbf{3 7}$.

2.5a Compound 30: Yield $0.082 \mathrm{~g}(94 \%$, pale yellow solid). M.p.: $210-212^{\circ} \mathrm{C}$; IR $\left(\mathrm{KBr}, \mathrm{cm}^{-1}\right) 3057,3030$, 2986, 2943, 2849, 1632, 1600, 1490, 1457, 1364, 975, 756. ${ }^{1} \mathrm{H}$ NMR $\left(400 \mathrm{MHz}, \mathrm{CDCl}_{3}\right) \delta 1.37-1.40(\mathrm{~m}, 2 \mathrm{H}$, cyclohexyl- $H$ ), 1.62 (br s, $2 \mathrm{H}$, cyclohexyl- $H$ ), 1.81$1.88(\mathrm{~m}, 4 \mathrm{H}$, cyclohexyl- $H), 2.81(\mathrm{t}, J(\mathrm{H}-\mathrm{H})=6.0 \mathrm{~Hz}$, $2 \mathrm{H}$, cyclohexyl- $H), 5.86(\mathrm{~d}, J(\mathrm{H}-\mathrm{H})=16 \mathrm{~Hz}, 1 \mathrm{H}$, $\left.\mathrm{CH}_{\mathrm{A}}=\mathrm{CH}_{\mathrm{B}}\right), 7.16-7.24(\mathrm{~m}, 5 \mathrm{H}, \operatorname{Ar}-H), 7.30(\mathrm{t}, J(\mathrm{H}-$ $\mathrm{H})=7.6 \mathrm{~Hz}, 2 \mathrm{H}, \operatorname{Ar}-H), 7.34-7.36(\mathrm{~m}, 2 \mathrm{H}, \operatorname{Ar}-H)$, $7.44\left(\mathrm{~d}, J(\mathrm{H}-\mathrm{H})=15.6 \mathrm{~Hz}, 1 \mathrm{H}, C \mathrm{H}_{\mathrm{A}}=\mathrm{C} H_{\mathrm{B}}\right), 7.59$ $7.63(\mathrm{~m}, 2 \mathrm{H}, \operatorname{Ar}-H), 8.61(\mathrm{~d}, J=8.0 \mathrm{~Hz}, 2 \mathrm{H}, \operatorname{Ar}-H)$. 
${ }^{13} \mathrm{C}$ NMR $\left(100 \mathrm{MHz}, \mathrm{CDCl}_{3}\right) \delta 26.3,27.0,27.9,30.4_{5}$, $30.5_{2}, 116.6$, 119.8, 121.6, 122.0, 126.6, 127.4, 127.7, $128.0,128.6,130.0,133.8,136.5,141.8,146.5,178.2$ $(C=\mathrm{O})$. HRMS (ESI) calcd. for $\mathrm{C}_{28} \mathrm{H}_{26} \mathrm{NO}\left[\mathrm{M}^{+}+\mathrm{H}\right]$ : 392.2014. Found: 392.2013.

2.5b Compound 31: Yield $0.074 \mathrm{~g}$ (84\%, pale yellow solid). M.p.: $170-172^{\circ} \mathrm{C}$; IR $\left(\mathrm{KBr}, \mathrm{cm}^{-1}\right) 3063$, 2959, 2921, 2855, 1627, 1600, 1479, 1457, 1359, 1293, 1162, 1041, 932, 751. ${ }^{1} \mathrm{H}$ NMR $\left(400 \mathrm{MHz}, \mathrm{CDCl}_{3}\right.$ ) $\delta$ 1.35-1.37 (m, 2H, cyclohexyl- $H$ ), 1.61 (br s, $2 \mathrm{H}$, cyclohexyl- $H$ ), 1.80-1.87 (m, 4H, cyclohexyl- $H$ ), 2.27 $\left(\mathrm{s}, 3 \mathrm{H}, \mathrm{CH}_{3}\right), 2.80(\mathrm{t}, J(\mathrm{H}-\mathrm{H})=6.2 \mathrm{~Hz}, 2 \mathrm{H}$, cyclohexyl$H), 5.85\left(\mathrm{~d}, J(\mathrm{H}-\mathrm{H})=15.6 \mathrm{~Hz}, 1 \mathrm{H}, \mathrm{CH}_{\mathrm{A}}=\mathrm{CH}_{\mathrm{B}}\right), 7.04$ $(\mathrm{d}, J(\mathrm{H}-\mathrm{H})=7.6 \mathrm{~Hz}, 2 \mathrm{H}, \operatorname{Ar}-H), 7.14(\mathrm{~d}, J=8.0 \mathrm{~Hz}$, $2 \mathrm{H}, \mathrm{Ar}-H), 7.30(\mathrm{t}, J(\mathrm{H}-\mathrm{H})=7.8 \mathrm{~Hz}, 2 \mathrm{H}, \mathrm{Ar}-H), 7.35-$ $7.41\left(\mathrm{~m}, 3 \mathrm{H}, C \mathrm{H}_{\mathrm{A}}=\mathrm{CH}_{\mathrm{B}}+\operatorname{Ar}-H\right), 8.01(\mathrm{~d}, J=$ $8.0 \mathrm{~Hz}, 2 \mathrm{H}, \operatorname{Ar}-H), 8.62$ (d, $J=8.0 \mathrm{~Hz}, 2 \mathrm{H}, \operatorname{Ar}-H)$. ${ }^{13} \mathrm{C}$ NMR $\left(100 \mathrm{MHz}, \mathrm{CDCl}_{3}\right) \delta 21.2,26.3,27.0,27.9$, $30.4,30.5,116.7,118.4,121.6,121.9,126.5,127.4$, 127.8, 129.3, 130.0, 133.8, 138.0. 141.8, 145.6, 178.2 $(C=\mathrm{O})$. HRMS (ESI) calcd. for $\mathrm{C}_{29} \mathrm{H}_{28} \mathrm{NO}\left[\mathrm{M}^{+}+\mathrm{H}\right]$ : 406.2171. Found: 406.2172.

2.5c Compound 32: Yield $0.085 \mathrm{~g}(91 \%$, pale yellow solid). M.p.: $110-112^{\circ} \mathrm{C}$; IR (KBr, $\left.\mathrm{cm}^{-1}\right)$ 3063, 2937 , 2855, 1737, 1638, 1600, 1507, 1485, 1457, 1364, 1178, 1030, 942, 751. ${ }^{1} \mathrm{H}$ NMR $\left(400 \mathrm{MHz}, \mathrm{CDCl}_{3}\right) \delta 1.35-$ 1.37 (m, 2H, cyclohexyl- $H$ ), 1.60 (br s, 2H, cyclohexyl$H), 1.78-1.86(\mathrm{~m}, 4 \mathrm{H}$, cyclohexyl- $H), 2.80(\mathrm{t}, J(\mathrm{H}-$ $\mathrm{H})=6.0 \mathrm{~Hz}, 2 \mathrm{H}$, cyclohexyl- $H), 3.75\left(\mathrm{~s}, 3 \mathrm{H}, \mathrm{OCH}_{3}\right)$, $5.82\left(\mathrm{~d}, J(\mathrm{H}-\mathrm{H})=15.6 \mathrm{~Hz}, 1 \mathrm{H}, \mathrm{CH}_{\mathrm{A}}=\mathrm{CH}_{\mathrm{B}}\right), 6.76(\mathrm{~d}$, $\left.J(\mathrm{H}-\mathrm{H})=15.6 \mathrm{~Hz}, 2 \mathrm{H}, \mathrm{CH}_{\mathrm{A}}=\mathrm{CH}_{\mathrm{B}}+\mathrm{Ar}-H\right), 7.18(\mathrm{~d}$, $J(\mathrm{H}-\mathrm{H})=8.7 \mathrm{~Hz}, 2 \mathrm{H}$, Ar- $H), 7.30(\mathrm{~d}, J=7.9 \mathrm{~Hz}, 2 \mathrm{H}$, Ar-H), $7.36(\mathrm{~d}, J=8.7 \mathrm{~Hz}, 2 \mathrm{H}, \operatorname{Ar}-H), 7.58-7.62(\mathrm{~m}$, $3 \mathrm{H}, \operatorname{Ar}-H), 8.61(\mathrm{~d}, J=8.0 \mathrm{~Hz}, 2 \mathrm{H}$, Ar- $H) .{ }^{13} \mathrm{C}$ NMR $\left(100 \mathrm{MHz}, \mathrm{CDCl}_{3}\right) \delta 26.3,27.0,27.9,30.4,30.5,55.3$, 114.0, 116.7, 117.8, 121.6, 121.9, 127.3, 127.9, 129.3, $129.5,133.7,141.8,145.0,159.5,178.2(C=\mathrm{O})$. HRMS (ESI) calcd. for $\mathrm{C}_{29} \mathrm{H}_{28} \mathrm{NO}_{2}\left[\mathrm{M}^{+}+\mathrm{H}\right]$ : 422.212 . Found: 422.2119.

2.5d Compound 33: Yield $0.065 \mathrm{~g}$ (68\%, yellow solid). M.p.: $190-192^{\circ} \mathrm{C}$; IR $\left(\mathrm{KBr}, \mathrm{cm}^{-1}\right) 3054,2926$, 2849, 1638, 1605, 1512, 1479, 1342, 1293, 1161, 1118, 942, 767. ${ }^{1} \mathrm{H}$ NMR $\left(400 \mathrm{MHz}, \mathrm{CDCl}_{3}\right) \delta 1.40-1.43(\mathrm{~m}$, $2 \mathrm{H}$, cyclohexyl- $H$ ), 1.61-1.67 (m, 2H, cyclohexyl- $H$ ), 1.85-1.94 $(\mathrm{m}, 4 \mathrm{H}$, cyclohexyl- $H), 2.85(\mathrm{t}, J(\mathrm{H}-\mathrm{H})=$ $6.2 \mathrm{~Hz}, 2 \mathrm{H}$, cyclohexyl- $H), 5.91(\mathrm{~d}, J(\mathrm{H}-\mathrm{H})=15.6 \mathrm{~Hz}$, $\left.1 \mathrm{H}, \mathrm{CH}_{\mathrm{A}}=\mathrm{CH}_{\mathrm{B}}\right), 7.28-7.33(\mathrm{~m}, 4 \mathrm{H}, \operatorname{Ar}-H), 7.37(\mathrm{~d}$, $J=8.8 \mathrm{~Hz}, 2 \mathrm{H}, \mathrm{Ar}-H), 7.59-7.64\left(\mathrm{~m}, 3 \mathrm{H}, C \mathrm{H}_{\mathrm{A}}=\right.$ $\left.\mathrm{C} H_{\mathrm{B}}+\mathrm{Ar}-H\right), 8.01(\mathrm{~d}, J=8.0 \mathrm{~Hz}, 2 \mathrm{H}, \mathrm{Ar}-H), 8.61-$ $8.63(\mathrm{~m}, 2 \mathrm{H}, \mathrm{Ar}-H) .{ }^{13} \mathrm{C} \mathrm{NMR}\left(100 \mathrm{MHz}, \mathrm{CDCl}_{3}\right)$ $\delta 26.2,27.1,28.0,30.7_{5}, 30.8_{0}, 116.2,118.2,121.6$, $121.9,122.1,123.9,124.2,127.0,127.4,127.6,133.9$, 141.6, 143.1, 146.9, 150.6, $178.1(C=\mathrm{O})$. HRMS (ESI) calcd. for $\mathrm{C}_{28} \mathrm{H}_{25} \mathrm{~N}_{2} \mathrm{O}_{3}\left[\mathrm{M}^{+}+\mathrm{H}\right]$ : 437.1865. Found: 437.1866 .

2.5e Compound 34: Yield 0.096 g (93\%, pale yellow solid). M.p.: $192-194^{\circ} \mathrm{C}$; IR $\left(\mathrm{KBr}, \mathrm{cm}^{-1}\right) 3052,2964$, 2921, 2849, 1638, 1594, 1480, 1364, 1293, 1162, 1074, 937, 674. ${ }^{1} \mathrm{H}$ NMR $\left(400 \mathrm{MHz}, \mathrm{CDCl}_{3}\right) \delta 1.37$ (br s, $2 \mathrm{H}$, cyclohexyl- $H$ ), 1.61 (br s, $2 \mathrm{H}$, cyclohexyl- $H$ ), 1.80 $1.88(\mathrm{~m}, 4 \mathrm{H}$, cyclohexyl- $H), 2.80(\mathrm{t}, J(\mathrm{H}-\mathrm{H})=6.0 \mathrm{~Hz}$, $2 \mathrm{H}$, cyclohexyl- $H), 5.80(\mathrm{~d}, J(\mathrm{H}-\mathrm{H})=16 \mathrm{~Hz}, 1 \mathrm{H}$, $\left.\mathrm{CH}_{\mathrm{A}}=\mathrm{CH}_{\mathrm{B}}\right), 7.10(\mathrm{~d}, J=8.0 \mathrm{~Hz}, 2 \mathrm{H}$, Ar- $H), 7.28-$ $7.35(\mathrm{~m}, 6 \mathrm{H}, \operatorname{Ar}-H), 7.42(\mathrm{~d}, J(\mathrm{H}-\mathrm{H})=15.6 \mathrm{~Hz}, 1 \mathrm{H}$, $\left.\mathrm{CH}_{\mathrm{A}}=\mathrm{CH}_{\mathrm{B}}\right), 7.59-7.63(\mathrm{~m}, 2 \mathrm{H}, \operatorname{Ar}-H), 8.61(\mathrm{~d}$, $J=7.6 \mathrm{~Hz}, 2 \mathrm{H}, \mathrm{Ar}-H) .{ }^{13} \mathrm{C} \mathrm{NMR}\left(100 \mathrm{MHz}, \mathrm{CDCl}_{3}\right)$ $\delta 26.3,27.0,30.0,30.5,30.6,116.5,120.5,121.7$, 122.0, 127.4, 127.5, 128.1, 128.7, 131.7, 133.8, 135.5, 141.7, 147.3, $178.1(C=\mathrm{O})$. HRMS (ESI) calcd. for $\mathrm{C}_{28} \mathrm{H}_{25} \mathrm{BrNO}\left[\mathrm{M}^{+}+\mathrm{H}\right]: 470.1119$ and 472.1119. Found: 470.1116 and 472.1097

2.5f Compound 35: Yield $0.071 \mathrm{~g}$ (77\%, pale yellow solid). M.p.: $120-122^{\circ} \mathrm{C}$; IR $\left(\mathrm{KBr}, \mathrm{cm}^{-1}\right) 3057$, 2921, 2849, 1638, 1600, 1479, 1457, 1364, 1293, 1266, 1172, 1085, 937, 762. ${ }^{1} \mathrm{H}$ NMR $\left(400 \mathrm{MHz}, \mathrm{CDCl}_{3}\right) \delta$ 1.37-1.43 (m, 2H, cyclohexyl- $H), 1.61-1.68(\mathrm{~m}, 2 \mathrm{H}$, cyclohexyl- $H$ ), 1.80-1.91 (m, 4H, cyclohexyl- $H$ ), 2.80 $(\mathrm{t}, J(\mathrm{H}-\mathrm{H})=6.2 \mathrm{~Hz}, 2 \mathrm{H}$, cyclohexyl- $H), 5.81(\mathrm{~d}, J(\mathrm{H}-$ $\left.\mathrm{H})=16 \mathrm{~Hz}, 1 \mathrm{H}, \mathrm{CH}_{\mathrm{A}}=\mathrm{CH}_{\mathrm{B}}\right), 7.15-7.20(\mathrm{~m}, 4 \mathrm{H}$, Ar- $H), 7.27-7.34(\mathrm{~m}, 4 \mathrm{H}, \operatorname{Ar}-H), 7.40(\mathrm{~d}, J(\mathrm{H}-\mathrm{H})=$ $\left.15.7 \mathrm{~Hz}, 1 \mathrm{H}, C \mathrm{H}_{\mathrm{A}}=\mathrm{C} H_{\mathrm{B}}\right), 7.59-7.63(\mathrm{~m}, 2 \mathrm{H}, \mathrm{Ar}-H)$, $8.61(\mathrm{~d}, J=8.0 \mathrm{~Hz}, 2 \mathrm{H}, \mathrm{Ar}-H) .{ }^{13} \mathrm{C}$ NMR $(100 \mathrm{MHz}$, $\left.\mathrm{CDCl}_{3}\right) \delta 26.3,27.0,27.9,30.5,30.6,116.5,120.4$, 121.7, 122.0, 127.5, 127.8, 128.6, 128.7, 133.5, 133.8, 135.0, 141.7, 147.2, 178.1 ( $C=\mathrm{O}$ ). HRMS (ESI) calcd. for $\mathrm{C}_{28} \mathrm{H}_{25} \mathrm{ClNO}\left[\mathrm{M}^{+}+\mathrm{H}\right]:$ : 426.1624. Found: 426.1626 .

2.5g Compound 36: Yield 0.045 g (49\%, pale yellow solid). M.p.: $257-259^{\circ} \mathrm{C}$; IR $\left(\mathrm{KBr}, \mathrm{cm}^{-1}\right) 3057$, 2975, 2926, 2849, 2219, 1638, 1600, 1479, 1457, 1359, 1288, 1178, 937, 745. ${ }^{1} \mathrm{H} \mathrm{NMR}\left(400 \mathrm{MHz}, \mathrm{CDCl}_{3}\right) \delta$ 1.40-1.43 (m, 2H, cyclohexyl- $H), 1.60-1.63(\mathrm{~m}, 2 \mathrm{H}$, cyclohexyl- $H$ ), $1.84-1.93$ (m, 4H, cyclohexyl- $H$ ), 2.83 
$(\mathrm{t}, J(\mathrm{H}-\mathrm{H})=6.1 \mathrm{~Hz}, 2 \mathrm{H}$, cyclohexyl- $H), 5.86(\mathrm{~d}, J(\mathrm{H}-$ $\left.\mathrm{H})=15.7 \mathrm{~Hz}, 1 \mathrm{H}, \mathrm{C} H_{\mathrm{A}}=\mathrm{CH}_{\mathrm{B}}\right), 7.29-7.33(\mathrm{~m}, 6 \mathrm{H}$, $\operatorname{Ar}-H), 7.50(\mathrm{~d}, J=8.3 \mathrm{~Hz}, 2 \mathrm{H}, \operatorname{Ar}-H), 7.55(\mathrm{~d}, J(\mathrm{H}-$ $\left.\mathrm{H})=15.7 \mathrm{~Hz}, 1 \mathrm{H}, C \mathrm{H}_{\mathrm{A}}=\mathrm{CH}_{\mathrm{B}}\right), 7.60-7.64(\mathrm{~m}, 2 \mathrm{H}$, Ar- $H), 8.60-8.63$ (m, 2H, Ar- $H) .{ }^{13} \mathrm{C}$ NMR $(100 \mathrm{MHz}$, $\left.\mathrm{CDCl}_{3}\right) \delta 26.2,27.0,28.0,30.68,30.73,110.8,116.2$, 118.2, 121.8, 122.0, 123.4, 127.0, 127.4, 127.6, 128.0, 132.3, 133.9, 141.1, 141.6, 149.9, $178.1(C=\mathrm{O})$. HRMS (ESI) calcd. for $\mathrm{C}_{29} \mathrm{H}_{25} \mathrm{~N}_{2} \mathrm{O}\left[\mathrm{M}^{+}+\mathrm{H}\right]: 417.1967$ Found: 417.1968.

2.5h Compound 37: Yield $0.063 \mathrm{~g}$ (56\%, red solid). M.p.: $190-192^{\circ} \mathrm{C}$; IR $\left(\mathrm{KBr}, \mathrm{cm}^{-1}\right) 3057,2931,2855$, 1627, 1606, 1480, 1457, 1359, 1266, 1162, 1107, 1047, 959, 806, 751. ${ }^{1} \mathrm{H}$ NMR $\left(400 \mathrm{MHz}, \mathrm{CDCl}_{3}\right) \delta 1.37$ (d, 2H, cyclohexyl- $H$ ), 1.61 (br s, $2 \mathrm{H}$, cyclohexyl- $H$ ), 1.78-1.86 (m, 4H, cyclohexyl- $H), 2.71(\mathrm{t}, 2 \mathrm{H}, J(\mathrm{H}-$ $\mathrm{H})=5.8 \mathrm{~Hz}$, cyclohexyl- $H), 3.85(\mathrm{~s}, 5 \mathrm{H}$, ferrocenyl$H), 4.21-4.18(\mathrm{~m}, 4 \mathrm{H}$, ferrocenyl- $H), 5.58(\mathrm{~d}, J(\mathrm{H}-$ $\left.\mathrm{H})=15.2 \mathrm{~Hz}, 1 \mathrm{H}, \mathrm{C} H_{\mathrm{A}}=\mathrm{CH}_{\mathrm{B}}\right), 6.92(\mathrm{~d}, J(\mathrm{H}-\mathrm{H})=$ $\left.15.2 \mathrm{~Hz}, 1 \mathrm{H}, \mathrm{CH}_{\mathrm{A}}=\mathrm{CH}_{\mathrm{B}}\right), 7.31(\mathrm{t}, J(\mathrm{H}-\mathrm{H})=7.4 \mathrm{~Hz}$, $2 \mathrm{H}, \operatorname{Ar}-\mathrm{H}), 7.40(\mathrm{~d}, J=8.4 \mathrm{~Hz}, 2 \mathrm{H}, \mathrm{Ar}-H), 7.62-$ $7.66(\mathrm{~m}, 2 \mathrm{H}$, Ar- $H$ ), 8.63 (d, $J=8.0 \mathrm{~Hz}, 2 \mathrm{H}$, Ar- $H$ ). ${ }^{13} \mathrm{C}$ NMR $\left(100 \mathrm{MHz}, \mathrm{CDCl}_{3}\right) \delta 26.4,26.9,27.8,30.3$, $30.4,67.0,68.3,69.1,69.4,82.2,116.5,116.8,121.7$, 122.0, 127.4, 127.8, 129.4, 133.7, 141.8, 142.8, 178.3 $(C=\mathrm{O})$. HRMS (ESI) calcd. for $\mathrm{C}_{32} \mathrm{H}_{30} \mathrm{NOFe}\left[\mathrm{M}^{+}+\mathrm{H}\right]$ : 500.1674. Found: 500.1675.

\section{$2.6 X$-ray structural analysis of 18-19, 24-25 and 27}

Single crystal X-ray diffraction data for compounds 18 and 24 were collected on a Bruker AXS-SMART diffractometer and that for 19, 25 and $27 \cdot 1 / 2 \mathrm{MeOH}$ were collected on an OXFORD diffractometer, using Mo $\mathrm{K}_{\alpha}(\lambda=0.71073 \AA)$ radiation. The structures were determined and refined by standard methods. ${ }^{15}$ In the structure of compound 18, the bromine at $\mathrm{C} 11$ and hydrogen at $\mathrm{C} 17$ exchanged positions, leading to partial occupancy at these positions while packing, but the structure was refined well. Full details of the $\mathrm{X}$-ray structure solution and refinement as a CIF file are available as Supplementary Information.

2.6a Compound 18: pale yellow block, $\mathrm{C}_{52} \mathrm{H}_{58} \mathrm{Br}_{2} \mathrm{~N}_{2} \mathrm{O}_{8} \mathrm{P}_{2}, M=1060.76$, triclinic, Space group $P \overline{1}, a=11.052(6), b=11.233(6), c=11.782(6) \AA$, $\alpha=98.582(8), \beta=106.272(7), \gamma=112.690(7)$, $V=1239.5(11) \AA^{3}, Z=1, \mu=1.755 \mathrm{~mm}^{-1}$, data/restraints/parameters: 4338/0/315, $\mathrm{R}$ indices $(\mathrm{I}>2 \sigma(\mathrm{I})): \mathrm{R} 1=0.0661, w \mathrm{R} 2($ all data $)=0.1637$. CCDC No. 942493. 2.6b Compound 19: pale brown block, $\mathrm{C}_{30} \mathrm{H}_{26} \mathrm{NO}_{2} \mathrm{P}$, $M=463.49$, Monoclinic, Space group $P 2_{1} / \mathrm{c}$, $a=11.072(2), b=16.360(3), c=15.858(4) \AA$, $\beta=121.423(15), V=2451.2(9) \AA^{3}, Z=4, \mu=$ $0.140 \mathrm{~mm}^{-1}$, data/ restraints/parameters: 4301/0/309, $\mathrm{R}$ indices $(\mathrm{I}>2 \sigma(\mathrm{I})): \mathrm{R} 1=0.0626, w \mathrm{R} 2$ (all data) $=$ 0.1858. CCDC No. 942496.

2.6c Compound 24: colourless block, $\mathrm{C}_{23} \mathrm{H}_{24} \mathrm{NO}_{3} \mathrm{P}$, $M=393.40$, Monoclinic, Space group $P 2 / \mathrm{c}$, $a=16.345(7), b=6.288(3), c=19.938(8) \AA, \beta=$ 96.169(7), $V=2037.3(15) \AA^{3}, Z=4, \mu=$ $0.158 \mathrm{~mm}^{-1}$, data/ restraints/parameters: $3597 / 0 / 257$, $\mathrm{R}$ indices $(\mathrm{I}>2 \sigma(\mathrm{I})): \mathrm{R} 1=0.0489, w \mathrm{R} 2($ all data $)=$ 0.1258. CCDC No. 942494.

2.6d Compound 25: colourless needles, $\mathrm{C}_{26} \mathrm{H}_{28} \mathrm{NO}_{3} \mathrm{P}$, $M=433.46$, Monoclinic, Space group $P 2_{1} / \mathrm{c}, a=$ 6.3172(7), $b=14.0629(16), c=27.295(3) \AA, \beta=$ 108.966(12), $V=2293.2(5) \AA^{3}, Z=4, \mu=0.147 \mathrm{~mm}^{-1}$, data/restraints/parameters: 3907/0/282, R indices (I > $2 \sigma(\mathrm{I})): \mathrm{R} 1=0.0583, w \mathrm{R} 2$ (all data) $=0.1090 . \mathrm{CCDC}$ No. 942495.

2.6e Compound 27.1/2MeOH: colourless blocks, $\mathrm{C}_{51} \mathrm{H}_{62} \mathrm{~N}_{8} \mathrm{O}_{9} \mathrm{P}_{2}, M=993.03$, triclinic, Space group $P \overline{1}$, $a=10.0712(12), b=11.645(2), c=11.8217(16) \AA$, $\alpha=85.489(12), \beta=67.870(12), \gamma=83.492(12)$, $V=1275.0(3) \AA^{3}, Z=1, \mu=0.149 \mathrm{~mm}^{-1}$, data/ restraints/parameters: 4473/327, $\mathrm{R}$ indices (I > $2 \sigma(\mathrm{I}))$ : $\mathrm{R} 1=0.0972, w \mathrm{R} 2$ (all data) $=0.3057$. The data quality was only moderate and hence the hydrogen atoms on the solvent were not modelled; however, the basic structure of the molecule was unambiguous. CCDC No. 946920 .

\section{Results and discussion}

The reaction of allenylphosphonates $\left(\mathrm{OCH}_{2} \mathrm{CMe}_{2} \mathrm{CH}_{2} \mathrm{O}\right)$ $\mathrm{P}(\mathrm{O}) \mathrm{CH}=\mathrm{C}=\mathrm{CR}^{1} \mathrm{R}^{2}\left\{\mathrm{R}^{1}=\mathrm{R}^{2}=\mathrm{Me}(\mathbf{1}), \mathrm{R}^{1}=\mathrm{R}^{2}=\right.$ $\left.\left[-\mathrm{CH}_{2-}\right]_{5}(\mathbf{2})\right\}$, with various substituted acridones $(\mathbf{1 0}-\mathbf{1 2})$ at $100^{\circ} \mathrm{C}$ in DMF solvent by using $\mathrm{CsF}$ as base leads to the formation of acridonyl allylphosphonates 13-18 (scheme 2). Although the reaction worked well even by using one mol equivalent of CsF, more equivalents were used to ensure the completion of reaction in a shorter period. The products were formed quantitatively $\left({ }^{31} \mathrm{P}\right.$ NMR) and the yields after isolation were $>80 \%$. By employing these conditions 


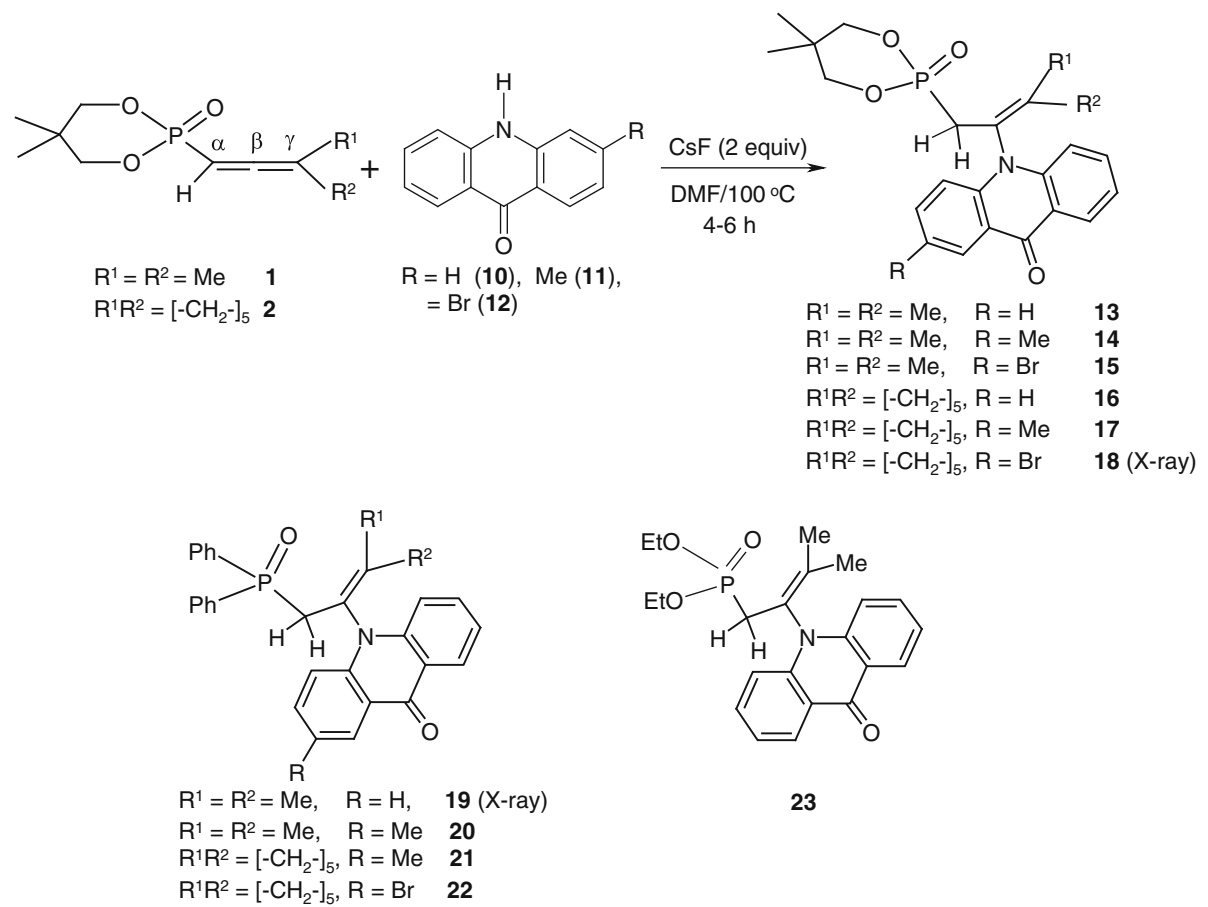

Scheme 2. Synthesis of acridonyl allylphosphonates/allylphosphine oxides 13-23.

to allenylphosphine oxides $\mathrm{Ph}_{2} \mathrm{P}(\mathrm{O}) \mathrm{C}(\mathrm{H})=\mathrm{C}=\mathrm{CR}^{1} \mathrm{R}^{2}$ $\left\{\mathrm{R}^{1}=\mathrm{R}^{2}=\mathrm{Me}(\mathbf{3}), \mathrm{R}^{1}=\mathrm{R}^{2}=\left[-\mathrm{CH}_{2^{-}}\right]_{5}(\mathbf{4})\right\}$ and the allenylphosphonate $(\mathrm{EtO})_{2} \mathrm{P}(\mathrm{O}) \mathrm{C}(\mathrm{H})=\mathrm{C}=\mathrm{CMe}(\mathbf{5})$, we could also obtain acridonyl allylphosphine oxides (19-22) and acridonyl allylphosphonate 23. The reaction did not occur in the absence of CsF (base); this observation is significant since normal amines or nucleobases do not require the presence of $\mathrm{CsF}^{4}$ Although we have not investigated this point in detail, it appears that the steric bulk and lower basicity of acridone requires fluoride activation.
In all the above compounds, the ${ }^{1} \mathrm{H}$ NMR spectra show a distinct peak in the region $\delta 3 \cdot 0-4.0[J(\mathrm{P}-$ $\mathrm{H}) \sim 21.0 \mathrm{~Hz}]$ indicating the presence of $\mathrm{PCH}_{2}$ group. Further confirmation of the structures is accomplished by the X-ray structure determination for compounds 18-19 (figure 1). The $\mathrm{C} 6-\mathrm{C} 7$ (in 18) or C13-C14 (in 19) distances are in the single bond range, as required.

It may be noted that in the above reactions, ${ }^{31} \mathrm{P}$ NMR spectrum of the reaction mixture shows the formation of a single product in each case. The other product (I) in
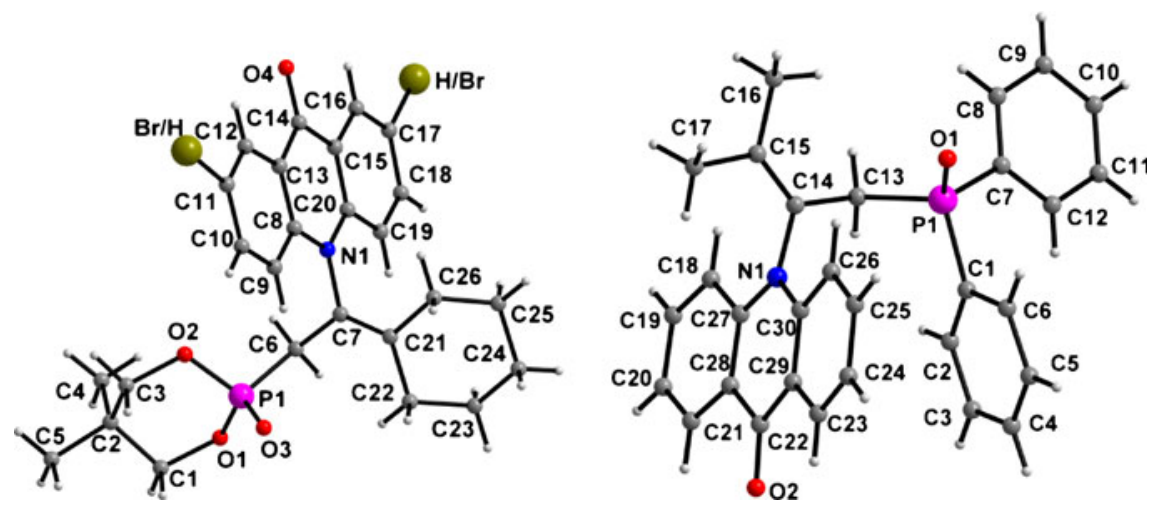

Figure 1. Molecular structures of 18 (left) and 19 (right). Selected bond distances are only given here. Compound 18 P1-C6 1.774(5), C6-C7 1.510(6), N1-C7 1.452(5) Å; compound 19 P1-C13 1.813(3), C13-C14 1.515(4), N1-C14 1.451(4) $\AA$. In the structure of compound 18, there is $\mathrm{Br} / \mathrm{H}$ exchange of positions at the carbon atoms $\mathrm{C} 11$ and $\mathrm{C} 17$. 
which the $\mathrm{N}-\mathrm{H}$ hydrogen is connected to the $\gamma$-carbon is not observed. Thus, the hydrogen moves regiospecifically to the carbon with the electron-withdrawing phosphoryl/phosphinoyl group.

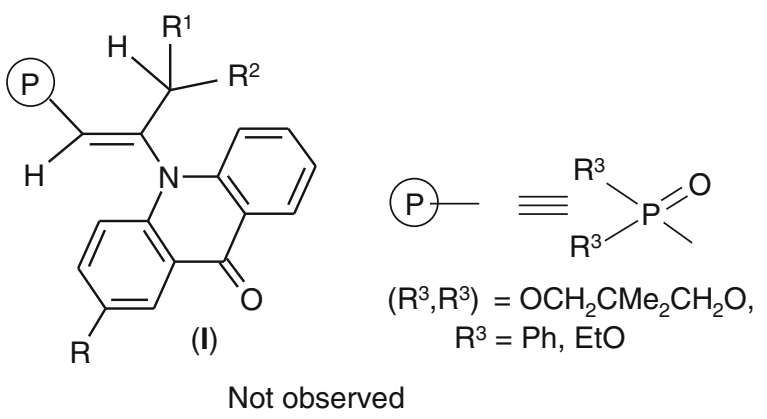

As mentioned in the Introduction, we initially intended to prepare acridinylphosphonates also by using [Pd]-catalysis. Thus, when compounds 1-2 were treated with 9-chloroacridine under the conditions $\mathrm{Pd}(\mathrm{OAc})_{2} / \mathrm{CsF} / \mathrm{DMF}$, we obtained two products in each case: Compound $\mathbf{1 3}$ (or 16) and the $\alpha$-substituted allene $\mathbf{2 4}$ (or 25). The products 13/16 must have arisen from the in situ formed acridone via adventitious moisture as encountered elsewhere. ${ }^{7}$ However, isolation of the $\alpha$-substituted products 24-25, albeit in ca 20\% yield was a surprise, because as mentioned in the Introduction (cf. scheme 1), the acridinyl residue should have gone to the $\beta$-position. Hence, we checked the reaction using 9-benzyl-6-chloropurine (26), since the $\mathrm{C}-\mathrm{Cl}$ bond in this compound is also fairly reactive. Although use of CsF did not work (no product) even after $24 \mathrm{~h}$, $\mathrm{Ph}_{3} \mathrm{P} / \mathrm{K}_{2} \mathrm{CO}_{3}$ as the base combination afforded the $\beta$ substituted product 27. ${ }^{16}$ This purinone product must have also formed in a manner similar to the acridone products 13 or 16 . The acridinyl and the acridone products $[\mathbf{1 3} / \mathbf{2 4}$ or $\mathbf{1 6} / \mathbf{2 5}]$ had essentially the same $R_{\mathrm{f}}$ value (posing difficulties in isolation) and hence the ${ }^{31} \mathrm{P} N M R$ had to be used as the diagnostic tool during elution.
As regards characterization of substituted allenylphosphonates $\mathbf{2 4 - 2 5},{ }^{13} \mathrm{C}$ NMR is quite useful. A distinct doublet at $c a \sim \delta 88\left[{ }^{1} J(\mathrm{P}-\mathrm{C})=197-199 \mathrm{~Hz}\right]$ is exhibited by the carbon $\alpha$ to the phosphorus. The large value of ${ }^{1} J(\mathrm{P}-\mathrm{C})$ indicates that the phosphorus is attached to an $\mathrm{sp}^{2}$ carbon. ${ }^{6}$ The ${ }^{31} \mathrm{P}$ NMR chemical shifts are in the region expected for such allenes and are close to those for 1-2. Final confirmation of the structures for 24-25 was accomplished by $\mathrm{X}$-ray crystallography (figure 2). The $\mathrm{C} 6-\mathrm{C} 7$ and $\mathrm{C} 7-\mathrm{C} 8$ distances (double bond range) as well as the C6-C7C8 bond angle clearly prove the allenic structure in both the cases. Steric factors during the formation of the $[\mathrm{Pd}]$-intermediate may be responsible for the formation of the $\alpha$-substituted products $\mathbf{2 4 / 2 5}$ in the case of reaction using 9-chloroacridne. The allylphosphonate $\mathbf{2 7}$ is 9-benzyl-purinone addition product with a purine nitrogen connected to $\beta$-carbon of the allene, but after the substitution of $-\mathrm{Cl}$ by $-\mathrm{OH}$ and rearrangement. For this compound, the $\mathrm{P}-\mathrm{CH}_{2}$ protons showed an AM pattern with coupling to phosphorus, thus exhibiting two doublets of doublet. More convincingly, the $\mathrm{P}-C$ carbon appeared in the aliphatic region $[\delta 30.4$, $\left.{ }^{1} J(\mathrm{P}-\mathrm{C})=94.0 \mathrm{~Hz}\right]$ which readily shows that this is an aliphatic carbon. The ${ }^{31} \mathrm{P}$ NMR chemical shift [ $\left.\delta 21.6\right]$ is also quite far from the precursor allenylphosphonate [ $\delta$ 10.0]; these data are consistent with the structure of 27 as proposed in scheme 3. X-ray structure was determined for $27 \cdot 1 / 2 \mathrm{MeOH}$ after crystallization from methanol (figure 3). The $\mathrm{P}-\mathrm{CH}_{2}$ moiety (at $\mathrm{C6}$ ) and the carbonyl group (at $\mathrm{C} 11$ ) are clearly discernible by the bond parameters associated with these atoms.

In the literature, it is reported that aniline undergoes $\mathrm{C}-\mathrm{H}$ activation and reacts with alkynes using $\mathrm{Pd}(\mathrm{OAc})_{2} /$ pivalic acid under aerobic conditions. ${ }^{17}$ Since the nitrogen of the acridone is connected to an aromatic carbon, we wanted to see if acridone can be
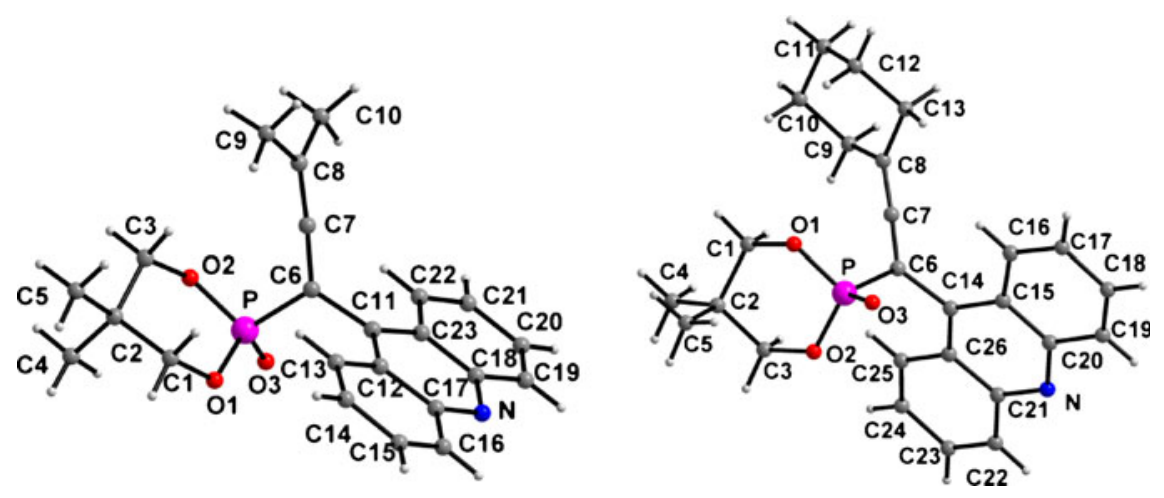

Figure 2. Molecular structures of $\mathbf{2 4}$ (left) and $\mathbf{2 5}$ (right). Selected bond parameters: Compound 24 P-C6 1.794(2), C6-C7 1.306(3), C7-C8 1.294(3) A, C6-C7C8 175.2(2) ${ }^{\circ}$; Compound 25 P-C6 1.798(4), C6-C7 1.304(5), C7-C8 1.283(5) A,, C6-C7-C8 173.6(5) . 
(a)

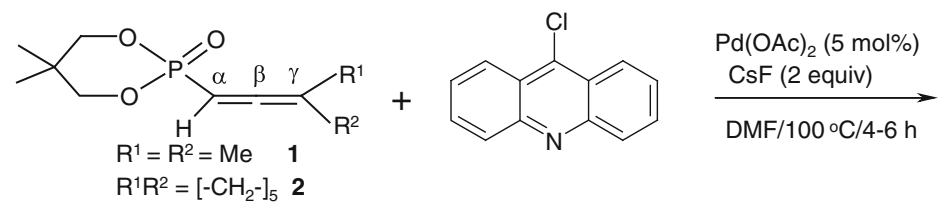<smiles>C=CC=C(CP1(=O)OCC(C)(C)CO1)n1c2ccccc2c(=O)c2ccccc21</smiles><smiles>[R]C([R])=C(c1c2ccccc2nc2ccccc12)P(=O)(OC)OCC(C)(C)C</smiles>

$\mathrm{R}^{1}, \mathrm{R}^{2}=\mathrm{Me} \quad 13$ $\mathrm{R}^{1} \mathrm{R}^{2}=\left[-\mathrm{CH}_{2}-\right]_{5} 16$

$\begin{array}{ll}\mathrm{R}^{1}, \mathrm{R}^{2}=\mathrm{Me} & \mathbf{2 4} \text { (X-ray) } \\ \mathrm{R}^{1} \mathrm{R}^{2}=\left[-\mathrm{CH}_{2}{ }^{-}\right]_{5} & 25 \text { (X-ray) }\end{array}$

(b)

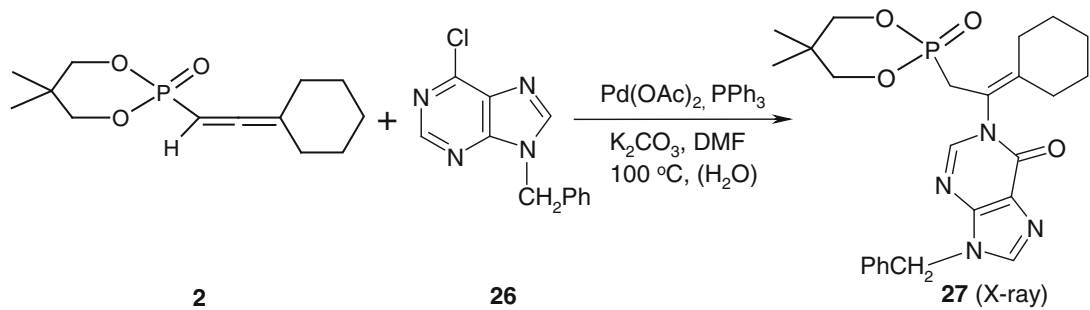

Scheme 3. [Pd]-catalysed reaction of allenylphosphonates 1-2 with 9-chloroacridine or 9-benzyl-6-chloropurine.

activated in a similar manner. However, no such activation occurred and acridone also did not add to the allene. Rather the rearranged product, the 1,3-butadiene 28 was obtained probably via an allylic [Pd] intermediate of type II (scheme 4); subsequently the strong conjugate base, pivalate anion, will abstract a proton from a terminal methyl group and the [Pd]-catalyst is released to form the product $\mathbf{2 8}$. Although there is a second possible structure for $\mathbf{2 8 - 2 9}$, the ${ }^{3} J_{\text {trans }}(\mathrm{HH})$ value is more consistent with the proposed one. It is also worthwhile to note that in the absence of pivalic acid only traces of the product were observed. In a similar manner, allene $\mathbf{2}$ gave the 1,3-butadiene 29. Here acridone is not involved in the reaction and hence it is possible to obtain the 1,3-butadienes $\mathbf{2 8}-\mathbf{2 9}$ in the absence of acridone. ${ }^{14}$

Compounds 13-23 possess a $\mathrm{P}(\mathrm{O}) \mathrm{CH}_{2}$ - group and hence we surmised that these would be attractive precursors for Horner-Wadsworth-Emmons (HWE) reaction. ${ }^{18}$ Initially, we used $t$-BuOK for the reaction using allyl acridone 16 but under these conditions, there was no reaction with acridone. Use of $\mathrm{NaH}$ as the base afforded the HWE products 30-36 readily in good yields (scheme 5). Even ferrocene carboxaldehyde reacted readily to afford the acridone derivative 37. Aromatic aldehydes containing electron-releasing $[\mathrm{Me}, \mathrm{OMe}]$ or electron-withdrawing groups $\left[\mathrm{NO}_{2}, \mathrm{Br}\right.$,

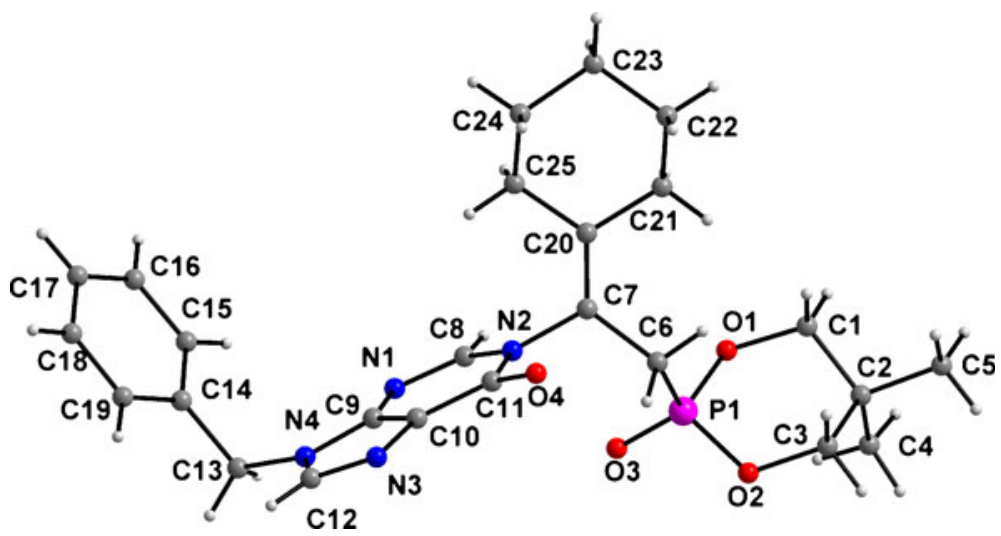

Figure 3. Molecular structure of $27 \cdot 1 / 2 \mathrm{MeOH}$. The solvent molecule is not shown. Selected bond parameters: P1-C6 1.764(5), C6-C7 1.497(7), N2-C7 1.454(5) Å, C6-C7-N2 113.0(4) ${ }^{\circ}$. 


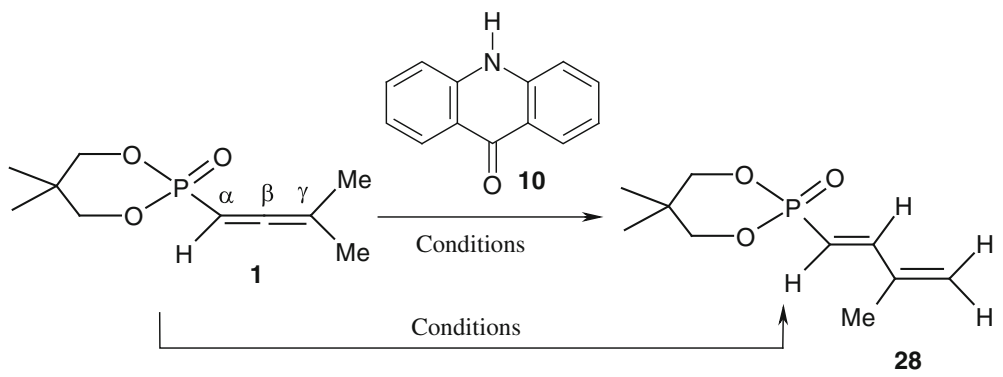

Conditions: $\mathrm{Pd}(\mathrm{OAc})_{2} / \mathrm{DMF}$ (or DMA):pivalic acid $(4: 1) / 120^{\circ} \mathrm{C}, 1 \mathrm{~h}$
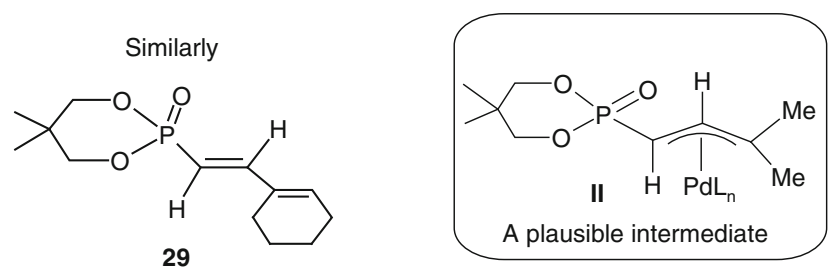

Scheme 4. [Pd]-catalysed rearrangement of allenylphosphonate 1-2 to phosphonobutadienes 28-29.

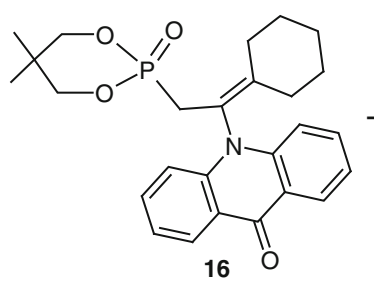

Sim<smiles>CCc1ccc(C)cc1</smiles><smiles>N#CC(=O)C(C=O)C(=O)O</smiles>

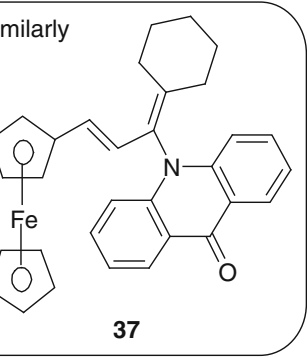

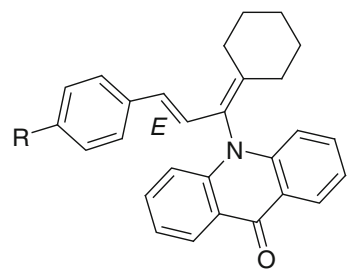

$\mathrm{R}=\mathrm{H} \quad 30$

$=\mathrm{Me} \quad 31$

$=\mathrm{OMe} \quad 32$

$=\mathrm{NO}_{2} \quad 33$

$=\mathrm{Br} \quad 34$

$=\mathrm{Cl} \quad 35$

Scheme 5. Horner-Wadsworth-Emmons reaction of $\mathbf{1 6}$ with aldehydes leading to the acridonyl-1,3-butadienes 30-37.

$\mathrm{Cl}$ and $\mathrm{CN}$ ] worked well. The $(E)$-configuration at the newly formed double bond is corroborated by the corresponding ${ }^{3} J(\mathrm{HH})$ values as well as the literature. ${ }^{18}$

\section{Summary}

Simple base-mediated (CsF) addition of acridone to allenylphosphonates/allenylphosphine oxides leads to acridonyl allylphosphonates wherein the nitrogen of the acridone is bonded to the carbon $\beta$ to phosphorus and the corresponding $\mathrm{N}-\mathrm{H}$ hydrogen moves to the $\alpha$-carbon. These compounds are good substrates for HWE reaction and are useful in generating a new class of $N$-substituted acridones. In two examples studied,
[Pd]-catalysed reaction of the allenylphosphonates led to the acridonyl allylphosphonates and the rather unexpected $\alpha$-substituted allenylphosphonates. In the presence of a carboxylic (pivalic) acid and $\mathrm{Pd}(\mathrm{OAc})_{2}$, allenylphosphonates with a terminal $=\mathrm{CR}_{2}[\mathrm{R}=\mathrm{Me}$; $\mathrm{R}_{2}=$ cyclohexyl] group undergo facile rearrangement to 1,3-butadienyl substituted phosphonates.

\section{Supplementary information}

The electronic supporting information (CIF file containing the details of crystal structures of compounds reported in this work) can be seen in www.ias.ac.in/chemsci. 


\section{Acknowledgements}

We thank the Department of Science and Technology (DST), New Delhi for financial support and for setting up of the National Single Crystal Diffractometer Facility at the University of Hyderabad. We also thank University with Potential for Excellence (UPE) programme of the University Grants Commission (UGC), New Delhi for equipment. KCK thanks DST for JC Bose fellowship and ALSK thanks UGC for a fellowship.

\section{References}

1. Selected reviews: (a) Hoffmann-Röder A and Krause N 2004 Angew. Chem. Int. Ed. 43 1196; (b) Pacheco M C, Purser S and Gouverneur V 2008 Chem. Rev. 108 1943; (c) Brasholz M, Reissig H-U and Zimmer R 2009 Acc. Chem. Res. 42 45; (d) Alcaide B, Almendros P and Aragoncillo C 2010 Chem. Soc. Rev. 39 783; (e) Yu S and Ma S 2012 Angew. Chem. Int. Ed. 513074

2. Selected recent references: (a) Zhou C, Fang Z, Fu C and Ma S 2009 J. Org. Chem. 74 2887; (b) Hirata Y, Inui T, Nakao Y and Hiyama T $2009 \mathrm{~J}$. Am. Chem. Soc. 131 6624; (c) Xu C F, Xu M, Yang L Q and Li C Y 2012 J. Org. Chem. 77 3010; (d) Hossain M L, Ye F, Zhang Y and Wang J 2013 J. Org. Chem. 781236

3. (a) Nishimura T, Hirabayashi S and Hayashi T 2006 J. Am. Chem. Soc. 126 2556; (b) Bravo-Altamirano K, Abrunhosa-Thomas I and Montchamp J L 2008 J. Org. Chem. 73 2292; (c) Chakravarty M, Bhuvan Kumar N N, Sajna K V and Kumara Swamy K C 2008 Eur. J. Org. Chem. 4500

4. Kumara Swamy K C, Balaraman E and Satish Kumar N 2006 Tetrahedron $\mathbf{6 2} 10152$

5. Hydroamination of allenes with amines: (a) Hill A W, Elsegood M R J and Kimber M C 2010 J. Org. Chem. 75 5406; (b) Cooke M L, Xu K and Breit B 2012 Angew. Chem. Int. Ed. 51 10876; (c) Butler K L, Tragni M and Widenhoefer R S 2012 Angew. Chem. Int. Ed. 51 5175

6. (a) Chakravarty M and Kumara Swamy K C 2006 J. Org. Chem. 71 9128; (b) Phani Pavan M, Chakravarty M and Kumara Swamy K C 2009 Eur. J. Org. Chem. 5927; (c) Bhuvan Kumar N N, Nagarjuna Reddy M and Kumara Swamy K C 2009 J. Org. Chem. 74 5395; (d) Phani Pavan M and Kumara Swamy K C 2011 Synlett 1288; (e) Rama Suresh R and Kumara Swamy K C 2012 J. Org. Chem. 776959

7. In the preparation of bisphosphonates bearing an acridine as core moiety by hydrophosphonylation of 9chloroacridine also, facile formation of acridones was noticed earlier. See: Srinivas V and Kumara Swamy K C 2009 ARKIVOC (xii) 31

8. See, for example: (a) Sánchez I, Reches R, Caignard D H, Renard P and Pujol M D 2006 Eur. J. Med. Chem.
41 340; (b) Goodell J R, Ougolkov A V, Hiasa H, Kaur H, Remmel R, Billadeau D D, Ferguson D M 2008 J. Med. Chem. 51179

9. (a) Kumar R and Kumari M 2011 J. Chem. Pharm. Res. 3 217; (b) Kelly J X, Smilkstein M J, Brun R, Wittlin S, Cooper R A, Lane K D, Janowsky A, Johnson R A, Dodean R A, Winter R, Hinrichs D J and Riscoe M K 2009 Nature 459 270; (c) Kelly J X, Smilkstein M J, Cooper R A, Lane K D, Johnson R A, Janowsky A, Dodean R A, Hinrichs D J, Winter R and Riscoe M 2007 Antimicrob. Agents Chemother. 51 4133; (d) Sepulveda C S, Garcia C C, Fascio M L, D’ Accorso N B, Docampo Palacios M L, Pellon R F and Damonte E B 2012 Antiviral Res. 93 62; (e) Tabarrini O, Manfroni G, Fravolini A, Cecchetti V, Sabatini S, Clercq E D, Rozenski J, Canard B, Dutartre H, Paeshuyse J and Neyts J 2006 J. Med. Chem. 49 2621; (f) Hernandez-Olmos V, Abdelrahman A, El-Tayeb A, Freudendahl D, Weinhausen S and Müller C E 2012 J. Med. Chem. 55 9576; (g) Lin Y-C and Chen C-T 2009 Org. Lett. 11 4858; (h) Putic A, Stecher L, Prinz H and Muller K 2010 Eur. J. Med. Chem. 455345

10. For another approach to $N$-substituted acridones, see: Singh P, Kaur J, Yadav B and Komath S S 2009 Bioorg. Med. Chem. 173973

11. Perrin D D, Armarego W L F and Perrin D 1986 Purification of laboratory chemicals (Oxford UK: Pergamon)

12. (a) Guillemin J C, Savignac P and Denis J M 1991 Inorg. Chem. 30 2170; (b) Iorga B, Eymery F, Carmichael D and Savignac P 2000 Eur. J. Org. Chem. 3103; (c) Guo H, Qian R, Guo Y and Ma S 2008 J. Org. Chem. 737934

13. (a) Allen C F H and McKee G H W 1939 Org. Synth. 19 6; (b) Rouband G, Faure R and Galy J-P 2003 Magn. Reson. Chem. 41549

14. Phani Pavan M 2010 New phosphono-heterocycles through allenylphosphonates, $\mathrm{PhD}$ thesis, University of Hyderabad

15. (a) Sheldrick G M 1996 SADABS, Siemens Area Detector Absorption Correction, University of Göttingen, Göttingen, Germany; (b) Sheldrick G M 1997 SHELX97, A Program for Crystal Structure Solution and Refinement, University of Göttingen, Göttingen, Germany; (c) Sheldrick G M 1999 SHELXTL NT Crystal Structure Analysis Package, Bruker AXS, Analytical X-ray System, Madison WI, version 5.10

16. Use of $\mathrm{Ph}_{3} \mathrm{P} / \mathrm{K}_{2} \mathrm{CO}_{3}$ condition did not work for 9chloroacridine

17. Shi Z, Zhang C, Li S, Pan D, Ding S, Cui Y and Jiao N 2009 Angew. Chem. Int. Ed. 484572

18. See, for example: (a) Abiko A and Masamune S 1996 Tetrahedron Lett. 37 1077; (b) Muthiah C, Praveen Kumar K, Aruna Mani C and Kumara Swamy K C 2000 J. Org. Chem. 65 3733; (c) Chakravarty M, Srinivas B, Muthiah C and Kumara Swamy K C 2003 Synthesis 2368 